\title{
Soluble epoxide hydrolase promotes astrocyte survival in retinopathy of prematurity
}

\author{
Jiong Hu, ${ }^{1,2}$ Sofia-Iris Bibli, ${ }^{1,2}$ Janina Wittig, ${ }^{1,2}$ Sven Zukunft, ${ }^{1,2}$ Jihong Lin, ${ }^{3}$ Hans-Peter Hammes, ${ }^{3}$ \\ Rüdiger Popp, ${ }^{1,2}$ and Ingrid Fleming ${ }^{1,2}$ \\ IInstitute for Vascular Signalling, Centre for Molecular Medicine, Goethe University, Frankfurt am Main, Germany. ²German Centre for Cardiovascular Research (DZHK) partner site Rhein-Main, \\ Frankfurt am Main, Germany. ${ }^{3}$ Fifth Medical Department, University Medicine Mannheim, University of Heidelberg, Mannheim, Cermany.
}

\begin{abstract}
Polyunsaturated fatty acids such as docosahexaenoic acid (DHA) positively affect the outcome of retinopathy of prematurity (ROP). Given that DHA metabolism by cytochrome P450 and soluble epoxide hydrolase (sEH) enzymes affects retinal angiogenesis and vascular stability, we investigated the role of sEH in a mouse model of ROP. In WT mice, hyperoxia elicited tyrosine nitration and inhibition of sEH and decreased generation of the DHA-derived diol 19,20-dihydroxydocosapentaenoic acid (19,20-DHDP). Correspondingly, in a murine model of $\mathrm{ROP}, \mathrm{sEH}^{-/-}$mice developed a larger central avascular zone and peripheral pathological vascular tuft formation than did their WT littermates. Astrocytes were the cells most affected by sEH deletion, and hyperoxia increased astrocyte apoptosis. In rescue experiments, 19,20-DHDP prevented astrocyte loss by targeting the mitochondrial membrane to prevent the hyperoxia-induced dissociation of presenilin-1 and presenilin-1-associated protein to attenuate poly ADP-ribose polymerase activation and mitochondrial DNA damage. Therapeutic intravitreal administration of

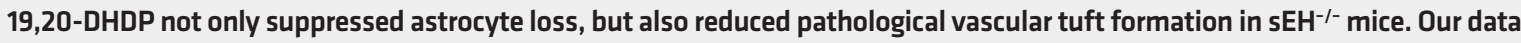
indicate that sEH activity is required for mitochondrial integrity and retinal astrocyte survival in ROP. Moreover, 19,20-DHDP may be more effective than DHA as a nutritional supplement for preventing retinopathy in preterm infants.
\end{abstract}

\section{Introduction}

Retinopathy of prematurity (ROP) is a complication of treating preterm infants with $\mathrm{O}_{2}$. It is estimated that as many as $10 \%$ of very premature infants become blind as a consequence of aberrant retinal neovascularization that leads to fibrovascular retinal detachment (1). Treatment strategies have focused on vascular ablative therapy and more recently on anti-VEGF-based approaches, but recurrence of disease and adverse effects are a cause of concern $(2,3)$. Another potential contributor to the disease is a deficiency of long-chain polyunsaturated fatty acids (PUFAs), particularly docosahexaenoic acid (DHA) (1,4), which is particularly abundant in the retina $(5,6)$. PUFAs such as DHA are fundamental structural components of neuronal and endothelial cells (7) and are required to maintain optimal retinal function. $\omega-3$ PUFAs are essential fatty acids and must be obtained through diet or total parenteral nutrition and are usually provided by the maternal diet during pregnancy, a transfer that cannot take place in preterm infants. Although dietary supplementation has shown some promise in preventing retinopathy (8) and has been linked with a coincident normalization of circulating adiponectin levels by modulating endoplasmic reticulum stress in white adipose tissue (9), exactly how the beneficial effects in the retina are achieved is unclear.

Authorship note: JH and SIB contributed equally to this work. Conflict of interest: The authors have declared that no conflict of interest exists. Copyright: () 2019, American Society for Clinical Investigation. Submitted: July 26, 2018; Accepted: August 28, 2019; Published: October 28, 2019 Reference information: J Clin Invest. 2019;129(12):5204-5218. https://doi.org/10.1172/JCl123835.
ROP can be mimicked in mice by exposing pups to $75 \% \mathrm{O}_{2}$ for 5 days - that is, from postnatal day (P) 7 until P12, and then returning them to room air for an additional 5 days before analysis on P17 (10). The initial period of hyperoxia results in the obliteration of vessels already formed in the central retina, which is then revascularized upon return to normal $\mathrm{O}_{2}$ levels (which results in a relative hypoxia in the retina), a process characterized by formation of preretinal vascular tufts, which reaches a maximum at P17 $(11,12)$. In this model, $\omega-3$ PUFAs (DHA and eicosapentaenoic acid) were able to directly suppress retinal neovascularization via a mechanism independent of VEGF (13). The link between DHA and ROP is particularly interesting because of the recent identification of cytochrome P450 (CYP) and soluble epoxide hydrolase $(\mathrm{sEH})$ enzymes in the retina that are able to metabolize DHA into fatty acid mediators, which can affect cell viability (14) and angiogenesis $(15,16)$. 19,20-dihydroxydocosapentaenoic acid (19,20-DHDP), a diol derived from DHA by the sequential action of CYP enzymes and sEH, was found to regulate angiogenesis by inhibiting the $\gamma$-secretase and Notch signaling (15). Therefore, the aim of this study was to determine the consequences of $\mathrm{sEH}$ deletion on vaso-obliteration and revascularization in a mouse model of ROP and assess whether 19,20-DHDP could directly affect revascularization.

\section{Results}

ROP in WT and $s \mathrm{EH}^{-/-}$littermates. ROP was studied in WT mice and their sEH-deficient ( $\left.\mathrm{sEH}^{-/}\right)$littermates. Focusing initially on neovascularization in WT mice on P17, the characteristic "tufting" of hyperproliferative endothelial cells in the central region as well as in residual avascular zones was observed (Figure 1A). Signifi- 
A
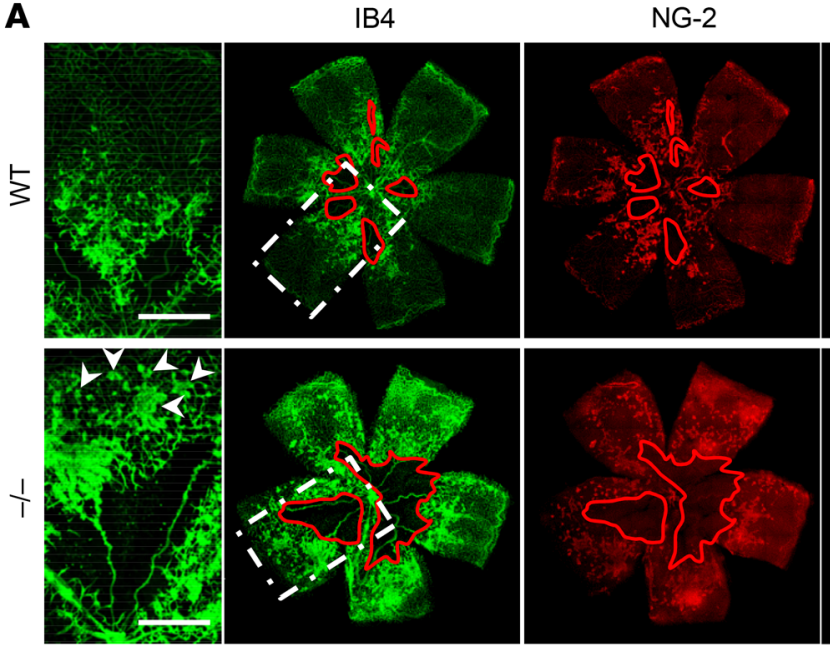

B
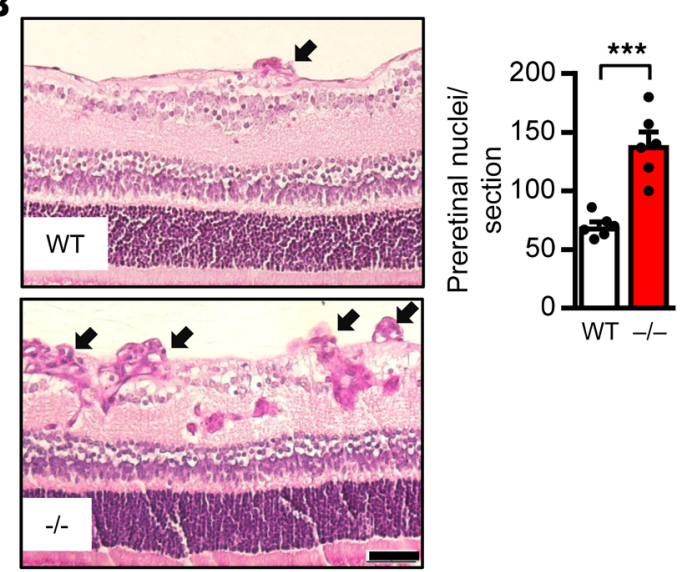

c

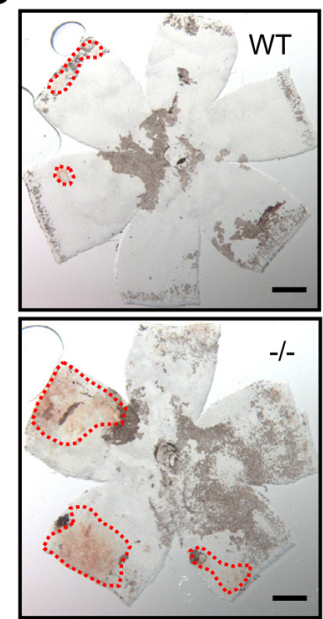

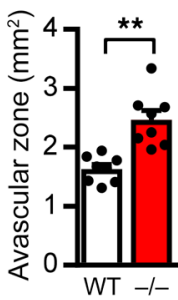
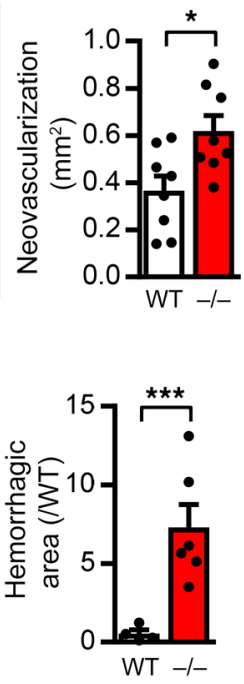

Figure 1. Comparison of the vascular phenotype in retinas from WT and sEH'/- mice with ROP. (A) Effect of sEH deletion on vascular ablation (avascular area) and neovascularization on P17. Endothelial cells were stained with isolectin B4 (IB4, green) and pericytes with NG-2 (red). Red lines indicate avascular zones. Arrows indicate peripheral neovascularization. Scale bar: $500 \mu \mathrm{m} . n=8$ animals/group. (B) Periodic acid Schiff and hematoxylin staining of cross-sections of retinas from WT and sEH $\mathrm{H}^{-1-}$ mice with ROP on P17. Periretinal nuclei above the inner limiting membrane are indicated by arrows. Scale bar: $50 \mu \mathrm{m} . n=6$ animals/group. (C) Whole mounts of retinas from WT and $\mathrm{sEH}^{-/-}$mice on P17. Regions with bleeding are highlighted with red dotted lines. Scale bars: $500 \mu \mathrm{m} . n=6$ animals/group. ${ }^{*} P<0.05$, ${ }^{* *} P<0.01$, and ${ }^{* *} P<0.001$ (Student's $t$ test).

cantly more vascular tufts and preretinal nuclei (Figure 1B) were evident in retinas from $\mathrm{sEH}^{-/}$mice. Unlike the samples from WT littermates, however, they were not restricted to the central region but also extended toward the periphery of the retina (see magnified area in Figure 1A). At the same time, there was a significantly larger avascular area as well as increased bleeding in the periphery of $\mathrm{sEH}^{-/}$retinas (Figure 1C), consistent with the fact that pathologic neovascularization results in the breakdown of the bloodretinal barrier and increases vascular leakage (11).

Consequence of $\mathrm{SEH}$ deletion on astrocyte survival. The increased avascular zone at P17 may be due to the increased vaso-obliteration during hyperoxia, a defect in vessel regrowth after the return of the animals to normoxia, or a combination of both. Therefore, to study the consequences of SEH deletion on vaso-obliteration, WT and $\mathrm{sEH}^{-/-}$littermates were exposed to hyperoxia for 24 hours (P8) or 5 days (P12). Under both conditions, a similar degree of vaso-obliteration was observed (Figure 2).
Retinal angiogenesis is closely linked to the underlying astrocyte scaffold (17), and to determine whether the defect in vessel (re) growth in $\mathrm{sEH}^{-/-}$retinas could be attributed to a defect at the level of astrocytes, a more detailed analysis was performed. Twentyfour hours after exposure to hyperoxia, glial fibrillary acidic protein (GFAP) staining was decreased in the central regions of WT retinas, an effect that was much more prominent in retinas from $\mathrm{sEH}^{-/-}$littermates (Figure 3A). A closer analysis of retinas on day 14 (i.e., 2 days after moving the mice from hyperoxia to normoxia) revealed alterations to the astrocyte network. In WT mice, there was a clear decrease in contact between astrocytes in the central area of the retina (Figure 3B), that was, again, more prominent in retinas from $\mathrm{sEH}^{-/-}$littermates. In the latter, there was also patchy astrocyte coverage of central retinal vessels and areas that were deficient in GFAP, indicating astrocyte loss. Interestingly, even at this early time point, more tuft-like structures were detected in the periphery of $\mathrm{sEH}^{-/-}$than WT retinas (see arrows in Figure 3B). In 

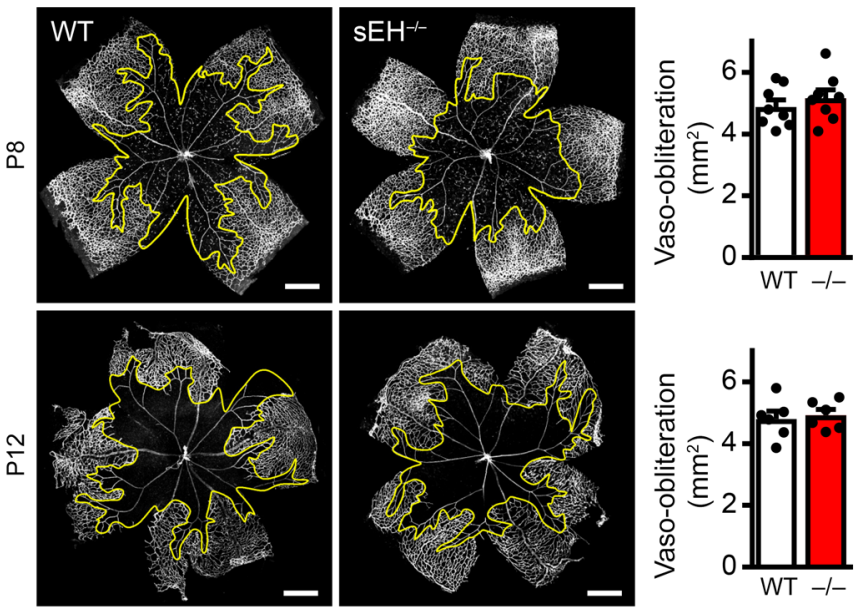

Figure 2. Consequences of sEH deletion on vaso-obliteration. Immunostaining of endothelial cells (isolectin B4) and quantification of the vaso-obliterated area in retinas from WT and $\mathrm{sEH}^{-/-}(-/-)$mice after exposure to hyperoxia for 1 or 5 days (P8 and P12, respectively). Yellow lines indicate the border of the vaso-obliterated region. Scale bars: $500 \mu \mathrm{m}$. $n=8$ for P8 and $n=6$ for P12.

line with previous studies indicating that astrocytes are vulnerable to hyperoxia (18), apoptosis (annexin V staining) was detected in GFAP-expressing cells in retinas from mice of both genotypes but was significantly greater in the $\mathrm{sEH}^{-/-}$group, as assessed by confocal imaging as well as FACS analysis (Figure 4, A and B). The role of $\mathrm{sEH}$ in astrocyte survival was studied on retinal astrocytes, which maintained sEH expression in culture (Figure 4C). Under normoxic conditions, fewer sEH-deficient than WT astrocytes were detected (Figure 4D), which correlated with a tendency $(P=0.1217)$ toward increased caspase activity (Figure $4 \mathrm{E})$. In response to hyperoxia, the numbers of $\mathrm{WT}$ and $\mathrm{sEH}^{-/}$astrocytes decreased, whereas caspase activity increased. Responses were significantly more marked, however, in the sEH-deficient group. VEGF plays a critical role in pathological neovascularization (19), and its expression was suppressed in retinas from mice exposed to hyperoxia for 24 hours (i.e., on P8) but increased on P14 during the relative hypoxic phase (Supplemental Figure 1; supplemental material available online with this article; https:/doi.org/10.1172/ JCI123835DS1). Although VEGF levels tended to be lower in samples from $\mathrm{sEH}^{-/-}$mice than in those from WT mice on P8, the differences were not significant.

In order to confirm the importance of $\mathrm{sEH}$ in astrocytes, we generated an astrocyte-specific sEH-knockout mouse line $\left(\mathrm{sEH}^{\triangle \mathrm{AC}}\right.$ ) by crossing $\mathrm{sEH}^{\mathrm{f} / \mathrm{fl}}$ mice with GFAP-Cre mice. Exposure of those animals to hyperoxia for 24 hours resulted in a similar degree of vaso-obliteration, but the astrocyte network was more profoundly affected in $\mathrm{sEH}^{\triangle \mathrm{AC}}$ mice than in their WT littermates (Figure 5, A and B). The astrocyte loss was reflected in a clear defect in revascularization on $\mathrm{P} 17$, as $\mathrm{sEH}^{\triangle \mathrm{AC}}$ mice showed a significantly larger avascular zone and increased unrestricted peripheral pathological vascular tuft formation (Figure 5, C and D).

Consequence of hyperoxia on sEH activity and retinal levels of 19,20-DHDP. sEH metabolizes PUFA epoxides to corresponding diols, therefore to determine whether the changes in astrocyte viability could be linked to the activity of sEH and the subsequent bioavailability of specific lipid metabolites, retinal PUFA epoxides and diols were assessed by liquid chromatography-tandem mass spectrometry (LC-MS/MS). In animals maintained under normoxic conditions, the levels of the arachidonic acid epoxides 11,12-epoxyeicosatrienoic acid (EET) and 14,15-EET were higher in retinas from $\mathrm{sEH}^{-/-}$versus WT mice (Figure 6A). The most pronounced changes were observed in retinal levels of the DHA diol 19,20-DHDP, however, which were lower in $\mathrm{sEH}^{-/}$retinas. In WT mice, hyperoxia $\left(75 \% \mathrm{O}_{2}, 24\right.$ hours) increased the levels of 8,9and 14,15-EET and decreased the levels of 11,12-dihydroxyeicosatrienoic acid (DHET) and 19,20-DHDP. There was no significant effect of hyperoxia on epoxide or diol levels in retinas from $\mathrm{sEH}^{-}$ mice. Given that hyperoxia did not alter the expression of either sEH or Cyp2c44 (Figure 6B), our data suggested that ROP was associated with $\mathrm{sEH}$ inhibition in retinas from WT mice and was confirmed in an sEH activity assay (Figure 6C).

Little is known about the mechanisms regulating the activity of sEH, but the active site of the enzyme contains 2 tyrosine residues (20) that can be nitrated to decrease enzymatic activity (21). This finding is of relevance, as hyperoxia has been reported to increase the expression of inducible nitric oxide synthase (iNOS) in the retina (22), particularly in GFAP-positive cells (23). Therefore, we addressed the hypothesis that hyperoxia inhibits $\mathrm{SEH}$ by inducing its tyrosine nitration. When $\mathrm{sEH}$ was immunoprecipitated from human embryonic kidney-293 (HEK-293) cells exposed to hyperoxia for 24 hours, its tyrosine nitration was increased (Figure 6D). The latter coincided with a significant reduction in sEH activity (Figure 6E). In line with the effects of hyperoxia observed in vitro, the sEH immunoprecipitated from WT retinas after a 24-hour exposure to hyperoxia was also tyrosine nitrated (Figure 6F). iNOS expression was also induced in the same retinas (Figure 6G) to act as a source of nitric oxide for the nitrosative stress induced by hyperoxia. Thus, although this study set out to compare ROP in WT and $\mathrm{sEH}^{-/-}$mice, the data generated indicate that in WT mice, the tyrosine nitration and inhibition of $\mathrm{sEH}$ is involved in the pathogenesis of the disease.

Effect of 19,20-DHDP supplementation on astrocyte loss. Given that hyperoxia markedly decreased retinal 19,20-DHDP levels in WT mice and levels were even lower in $\mathrm{sEH}^{-/-}$mice, we hypothesized that this PUFA diol may act as an astrocyte protective factor. To determine whether this was the case, the $\mathrm{sEH}$ substrate 19,20-epoxydocosapentaenoic acid (EDP) and the sEH product 19,20-DHDP were given intravitreally as a bolus (50 pmol) to $\mathrm{sEH}^{-/-}$mice immediately prior to placing them in hyperoxia for 24 hours. Pretreating mice with the PUFAs had no effect on early vaso-obliteration (Figure 7, A and B), but 19,20-DHDP was effective in preventing astrocyte loss (Figure 7, A and C). Similarly, the hyperoxia-induced increase in retinal annexin V in GFAP-positive cells was attenuated by 19,20-DHDP (Figure 7D). Given that 19,20-DHDP has been shown to inhibit $\gamma$-secretase-mediated Notch signaling in endothelial cells during retinal development (15), we determined if the effects observed could be attributed to the inhibition of Notch signaling. However, the intravitreal application of the $\gamma$-secretase inhibitor, $\mathrm{N}$-[N-(3,5-difluorophenacetyl)-L-alanyl]-S-phenylglycine t-butyl ester (DAPT), was not able to mimic the effects of 19,20-DHDP on astrocyte survival (Figure 
A

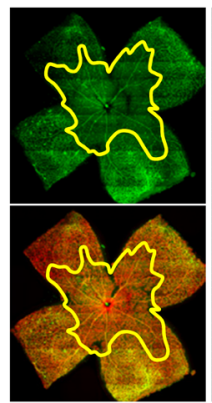

GFAP IB4

B
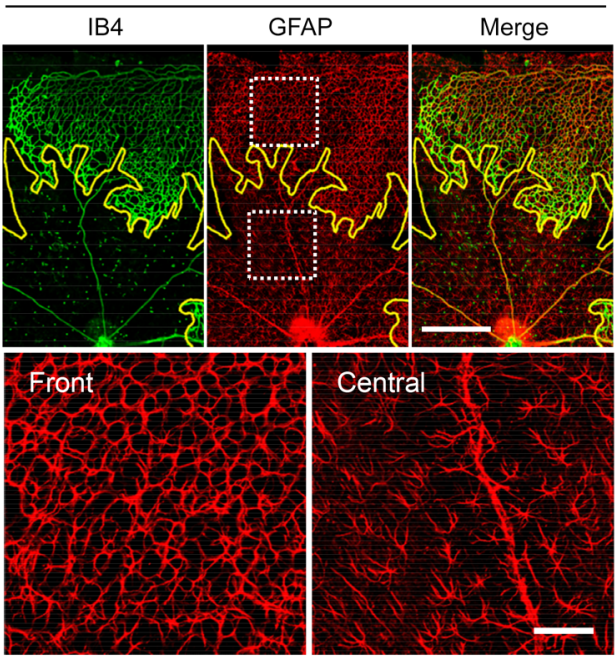
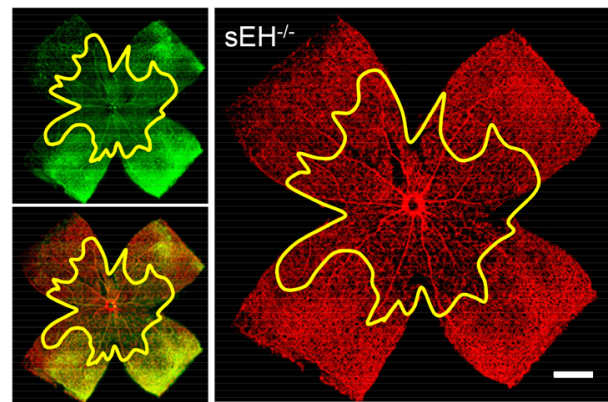

$\mathrm{sEH}^{-/-}$

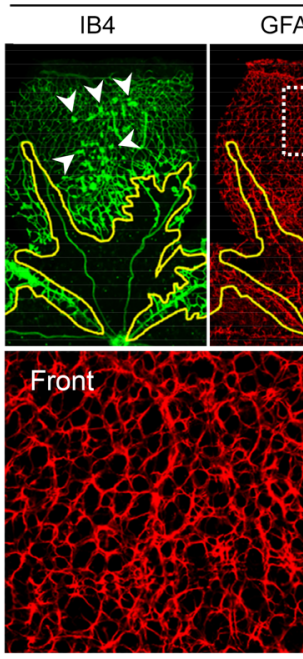

GFAP
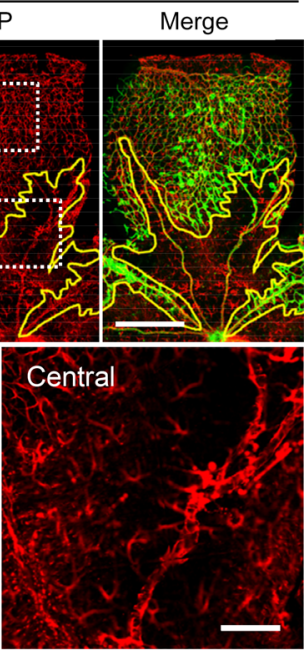
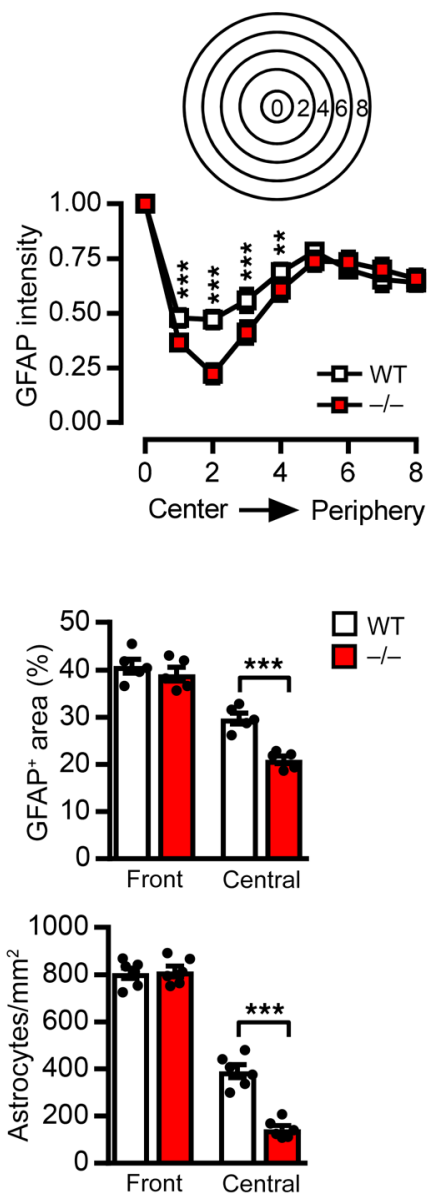

GFAP IB4

Figure 3. Consequences of sEH deletion on the astrocyte network. (A) Immunostaining of astrocytes (CFAP, red) and endothelial cells (isolectin B4, green) in $\mathrm{P} 8$ retinas from WT and $\mathrm{sEH}^{-/-}(-/-)$mice after exposure to hyperoxia for 1 day and quantification of GFAP intensity throughout the retina. The numbers 0 and 8 represent the optical nerve and retinal boundary, respectively. Scale bar: $500 \mu \mathrm{m}$. $n=8$ animals/group (2-way ANOVA and Sidak's multiple comparisons test). (B) Astrocytes (GFAP, red) and endothelial cells (isolectin B4, green) in retinas from WT and sEH ${ }^{-1-}$ mice on P14. Yellow lines highlight the border of avascular region. Arrows indicate peripheral neovascularization. Scale bar: $500 \mu \mathrm{m}$. The lower panels are higher-magnification images in the peripheral and central regions in retinas indicated by the boxes. Scale bar: $100 \mu \mathrm{m} . n=6$ animals/group with 8 areas of interest analyzed per retina (2-way ANOVA and Sidak's multiple comparisons test). ${ }^{* *} P<0.01$ and ${ }^{*}{ }^{*} P<0.001$.

7, A-D), indicating that 19,20-DHDP protects astrocytes against hyperoxia in a $\gamma$-secretase-independent manner. This protective effect was not limited to $\mathrm{sEH}^{-/-}$mice, as 19,20-DHDP was also able to protect against astrocyte loss in WT animals (Figure 7, E-G).

Effect of 19,20-DHDP on mitochondria potential and presenilin-1 (PS-1)-associated protein translocation. Cell death is commonly associated with, or initiated by, mitochondrial dysfunction and disruption of the mitochondrial transmembrane potential (24). Astrocytes from $\mathrm{sEH}^{--}$mice were more susceptible to hyperoxia-induced changes in the mitochondrial membrane potential than astrocytes from WT littermates, a phenomenon that was reversed following the addition of 19,20 DHDP but not 19,20-EDP (Figure 8A). Little is known about the effects of PUFA diols on organelle membrane integrity, but our previous observations $(15,25)$ indicated that 19,20-DHDP can influence membrane cholesterol distribution. This finding was relevant inasmuch as increased mitochondrial membrane cholesterol results in mitochondrial damage and death (26). Exposing HEK-293 cells to $75 \% \mathrm{O}_{2}$ for 48 hours significantly increased cholesterol levels in the mitochondrial membrane (Fig- ure $8 \mathrm{~B}$ ), and treating isolated mitochondria with cholesterol was coupled to a decrease in the mitochondrial membrane potential (Figure 8C).19,20-DHDP, but not 19,20-EDP, decreased cholesterol levels in the mitochondrial membrane and partially prevented the reduction in membrane potential triggered by cholesterol.

The cholesterol-interacting protein, PS-1, was previously characterized as a target of 19,20-DHDP $(15,25)$ and was of interest, as it has also been attributed to $\gamma$-secretase-dependent and -independent proapoptotic effects in mitochondria $(27,28)$. Given the lack of effect of DAPT on astrocyte loss in the mouse model, work focused on $\gamma$-secretase-independent actions of PS-1, which are mainly mediated by its interaction with the PS-1-associated protein (PSAP; also known as mitochondrial carrier homolog 1) (28). Under normoxic conditions, PS-1 and PSAP were colocalized in the same hydrophilic fractions (sucrose gradient) of the mitochondrial membrane (fractions 7-8) (Supplemental Figure 2, A and B). While PSAP was unaffected by exposure to hyperoxia for 24 hours, PS-1 was redistributed to more hydrophobic fractions (fractions $4-5)$, consistent with a greater association of PS-1 with cho- 
A

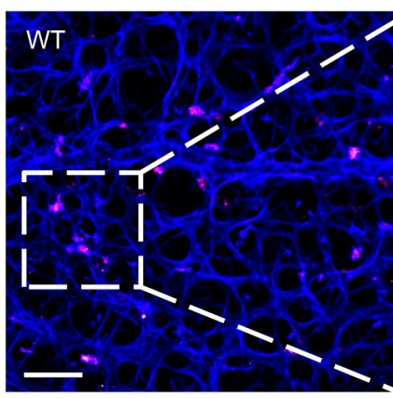

Annexin V GFAP
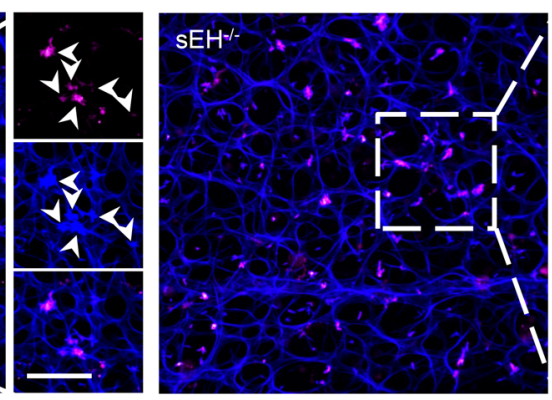

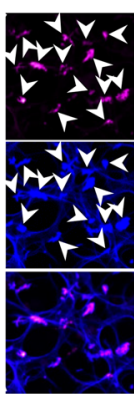

B

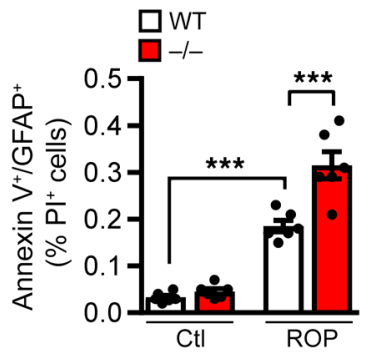

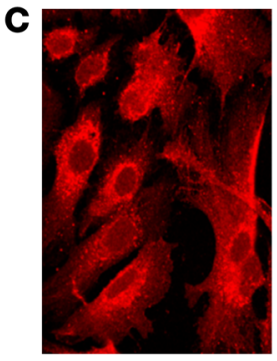

sEH GFAP DAPI
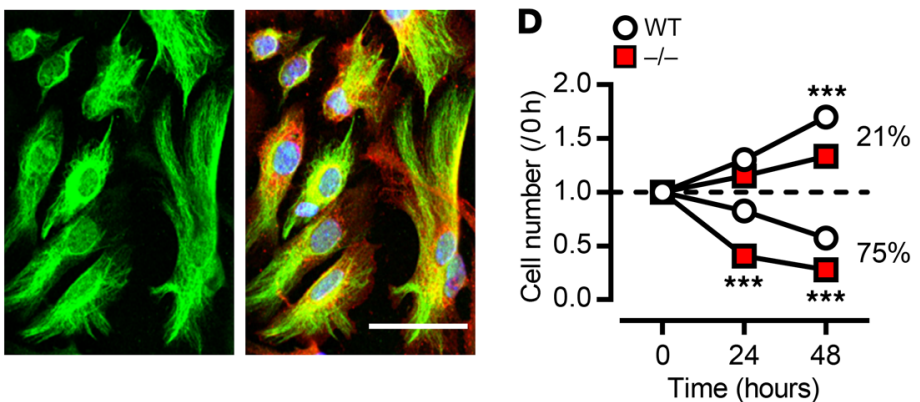

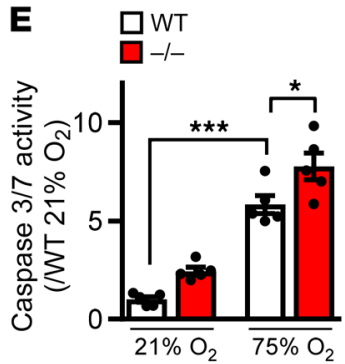

Figure 4. Link between sEH and astrocyte apoptosis. (A) Annexin V (magenta) and GFAP (blue) in retinal whole mounts from WT and sEH ${ }^{-1-}$ animals exposed to hyperoxia for 24 hours. Scale bars: $50 \mu \mathrm{m}$. Arrowheads indicate annexin $\mathrm{V}^{+} / G F A P^{+}$cells. $n=6$ animals/group. (B) Annexin $\mathrm{V}^{+} / G F A P^{+}$cells in retinal digests from WT or $\mathrm{SEH}^{-/-}$mice maintained under normoxic conditions or exposed to hyperoxia for 24 hours. $n=5$ animals/group (2-way ANOVA and Sidak's multiple comparisons test). Ctl, control. (C) sEH (red) and GFAP (green) expression in cultured primary retinal astrocytes. Scale bar: $50 \mu \mathrm{m}$. $n=$ 4 different cell batches. (D) Effect of $s$ EH deletion on primary astrocytes cultured under normoxic $\left(21 \% \mathrm{O}_{2}\right)$ or exposed to hyperoxia ( $\left.75 \% \mathrm{O}_{2}\right)$. $n=4$ different cell batches (2-way ANOVA and Sidak's multiple comparisons test). (E) Caspase $3 / 7$ activity in astrocytes under normoxic and hyperoxic conditions. $n=5$ different cell batches (2-way ANOVA and Sidak's multiple comparisons test). ${ }^{*} P<0.05$ and ${ }^{* * *} P<0.001$.

lesterol. When cholesterol was measured, there was a significant increase in cholesterol in fractions 4-5 from hyperoxia-treated mitochondria compared with those exposed to normoxia (Supplemental Figure 2C). Treating cells with 19,20-DHDP prevented the hyperoxia-induced redistribution of PS- 1 and cholesterol. In astrocytes cultured under normoxic conditions, PS-1 and PSAP could be coprecipitated, indicating a physical interaction (Figure 8D). Importantly, the interaction between PS- 1 and PSAP was attenuated in astrocytes exposed to $75 \% \mathrm{O}_{2}$. The dissociation of PS-1/ PSAP complex coincided with the translocation of PSAP from the outer to the inner mitochondrial membrane (Figure $8 \mathrm{E}$ ) and a significant increase in caspase 3 cleavage (Figure $8 \mathrm{~F}$ ). Treating astrocytes with 19,20-DHDP (but not 19,20-EDP) prevented the hyperoxia-induced changes in the association of the PS-1/PSAP complex as well as PSAP translocation and caspase 3 cleavage.

PS-1, PSAP, PARP1, and mitochondrial DNA damage. To link the in vitro observations back to ROP, experiments were repeated in retinas from WT and $\mathrm{sEH}^{-/}$mice exposed to hyperoxia for 24 hours. As in the in vitro studies, the interaction of PSAP with PS-1 was attenuated in hyperoxic $\mathrm{sEH}^{-/-}$retinas (Figure 9A) compared with the respective WT controls. While most of the cleaved caspase 3 in retinas from WT mice was detected in the outer nuclear layer with a subtle signal in astrocytes, there was a clear increase in caspase 3 cleavage in astrocytes in $\mathrm{sEH}^{-/-}$retinas (Figure 9B). The association of PSAP with PS-1 was also attenuated in SEH-deficient retinal astrocytes exposed to hyperoxia versus cells from WT mice (Figure 9C).

The proapoptotic actions of PSAP were previously linked to the activation of caspase 3 and the subsequent cleavage of PARP1 (29).
Interestingly, the exposure of WT mice to hyperoxia increased PARP1 cleavage in retinas from WT mice, while increased PARP1 cleavage was observed in retinas from $\mathrm{sEH}^{-/-}$mice under normoxic as well as hyperoxic conditions (Figure 9D). Following its cleavage, PARP1 DNA binding domains interact with mitochondria DNA (mtDNA) (30). Fitting with this, there was a detectable increase in mtDNA damage in normoxic $\mathrm{sEH}^{-/-}$versus WT retinas, which was further increased by exposure to hyperoxia (Figure 9E). Similarly, when retinal astrocytes were exposed to hyperoxia, PARP1 cleavage was significantly increased, and treatment with 19,20-DHDP (but not 19,20-EDP) prevented the hyperoxia-driven cleavage of PARP1 (Figure 9F). Moreover, chromatin immunoprecipitation studies showed that the binding of cleaved PARP1 to the D-loop and the ND2 domain of mtDNA was increased by hyperoxia by more than 100-fold (Figure 9G), as was mtDNA damage (Figure 9H). In line with the effect of 19,20-DHDP on PARP1 cleavage, the diol abolished mtDNA binding to the PARP1 DNA binding domain, as well as mtDNA damage in cultured astrocytes. To demonstrate the importance of PS-1, experiments were repeated using $\mathrm{sEH}^{-/}$ astrocytes treated with control oligonucleotides or small interfering (si) RNA directed against PS-1. The siRNA approach decreased astrocyte PS- 1 expression by $69 \pm 4 \%$ and prevented astrocyte loss in hyperoxic conditions. This was accompanied by reduced translocation of PSAP to the inner mitochondria membrane, reduced mtDNA damage, as well as decreased binding of PARP1 to mtDNA (Supplemental Figure 3). These findings highlighted the key role played by the interaction between PS-1 and PSAP in hyperoxiainduced astrocyte apoptosis. 
A
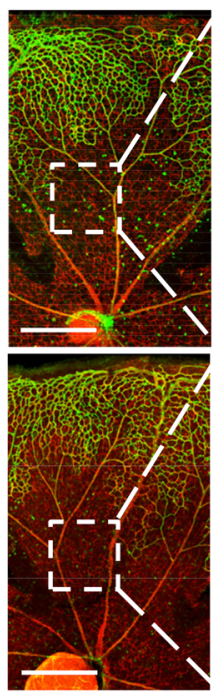

IB4 GFAP
B
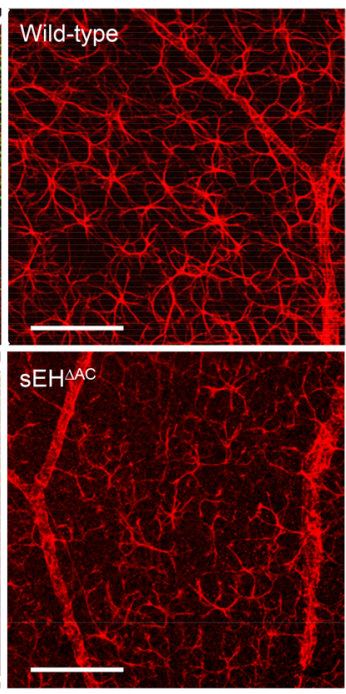

C
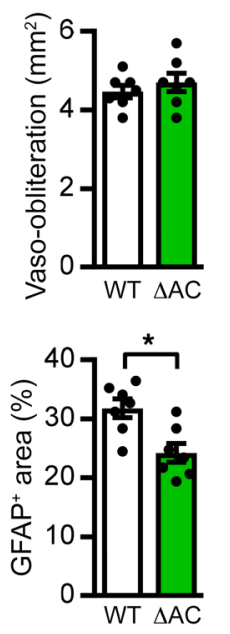
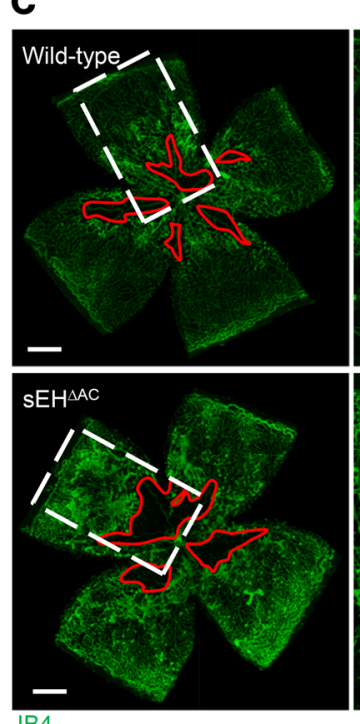
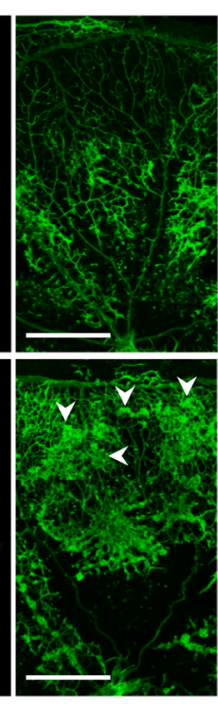

D
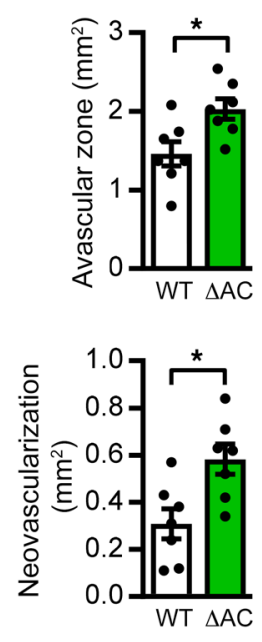

Figure 5. Phenotypes in astrocyte-specific sEH knockout mice. (A) Astrocytes (GFAP, red) and endothelial cells (isolectin B4, green) in retinas from WT and $\mathrm{sEH} \mathrm{HAC}^{\triangle \mathrm{AC}}$ littermates after exposure to $75 \% \mathrm{O}_{2}$ for 24 hours. Scale bars: $500 \mu \mathrm{m}$ in the whole mounts and $100 \mu \mathrm{m}$ for the enlarged images. $n=7$ animals $/$ group (ANOVA with Bonferroni's post test). (B) Quantification of vaso-obliteration and GFAP ${ }^{+}$area in retinas after exposure to $75 \% \mathrm{O}_{2} \mathrm{for}_{24}$ hours (Student's $t$ test). (C) Isolectin B4 staining in retinas from WT and sEH ${ }^{\triangle A C}$ littermates with ROP on P17. Red lines indicate the avascular area. Scale bars: 500 $\mu \mathrm{m}$ in the whole mounts and $100 \mu \mathrm{m}$ for the enlarged images. (D) Quantification of the avascular zone and neovascularization on P17. $n=7$ animals/group (Student's $t$ test). ${ }^{*} P<0.05$.

19,20-DHDP prevents neovascularization in ROP. To identify potential effects of 19,20-DHDP on mitochondrial integrity in ROP in vivo, 19,20-DHDP or its precursor epoxide 19,20-EDP were administered intravitreally to WT and $\mathrm{sEH}^{-/-}$littermates on P7 before the animals were exposed to hyperoxia for 24 hours. 19,20-DHDP (but not 19,20-EDP) was able to prevent the cleavage of both caspase 3 and PARP1 (Figure 10A) and prevent mtDNA damage (Figure 10B) in retinas from both genotypes. Finally, we asked whether 19,20-DHDP was able to rescue the retinopathy of the premature ROP phenotype. To assess this issue, $\mathrm{sEH}^{-/-}$mice were administered 19,20-EDP or 19,20-DHDP either immediately before being exposed to hyperoxia on $\mathrm{P} 7$, or immediately after their return to normoxia on P12 with vascularization being assessed on P17. Giving 19,20-DHDP on P7 to prevent astrocyte apoptosis clearly decreased the avascular zone as well as the number of vascular tufts detected on P17 when compared with retinas treated with solvent or 19,20-EDP (Figure 10C). Delaying 19,20-DHDP administration by 5 days was associated with a poorer revascularization of the retina, but the avascular area and the numbers of vascular tufts were still clearly less in animals that received 19,20-DHDP than in those that received 19,20-EDP or solvent (Figure 10D). We previously reported that high levels of 19,20-DHDP and the overexpression of sEH in Müller cells results in vascular destabilization and in retinopathy (16). As our data implied that low concentrations of 19,20-DHDP are vasoprotective while high concentrations elicit a deleterious effect, dose-response studies were performed in WT mice. Pups were treated intravitreally with increasing concentrations of 19,20-DHDP and vascularization. In addition, the astrocyte scaffold was assessed after 24 hours of exposure to hyperoxia (on P8) and following removal to room air for 5 days (i.e., on P17). In animals exposed to hyperox- ia for 24 hours, a bolus application of 19,20-DHDP did not affect vaso-obliteration, but it prevented astrocyte loss at concentrations up to and including 150 pmol (Supplemental Figure 4A). No benefit was seen using the highest concentration studied (i.e., 500 pmol). Also on day 17 , the avascular zone was significantly reduced in mice that were treated with 19,20-DHDP (Supplemental Figure $4 \mathrm{~B})$. However, while the avascular area and vascular tuft formation (neovascularization) were reduced by 50-pmol 19,20-DHDP, higher concentrations resulted in the appearance of avascular abnormalities characterized by tortuous vessels and the loss of the clear branching pattern at the periphery of the vascular plexus.

\section{Discussion}

The results of the present investigation revealed that the DHAderived diol, 19,20-DHDP, protects against astrocyte apoptosis and the development of ROP. Hyperoxia-induced astrocyte death in vivo and in vitro was linked to 19,20-DHDP-sensitive alterations in the cholesterol content of the astrocyte mitochondrial membrane, dissolution of the PS-1/PSAP complex, relocalization of PSAP to the inner mitochondrial membrane, caspase 3 activation, PARP1 cleavage, and mtDNA damage. Extrapolating the data further, it seems that the expression of sEH in the newborn retina protects against the development of ROP by generating 19,20DHDP. Inhibition of sEH may even, at least partly, underlie the pathology of the ROP, as retinal hyperoxia in WT mice resulted in the tyrosine nitration and inhibition of sEH.

Given the importance of DHA in the retina $(5,6)$, there have been a number of studies that have assessed potential benefits of dietary supplementation with $\omega-3$ PUFAs on the development of ocular diseases, including $\operatorname{ROP}(8,9,31)$. Although potential benefits of DHA therapy have been reported (32), it is unclear 

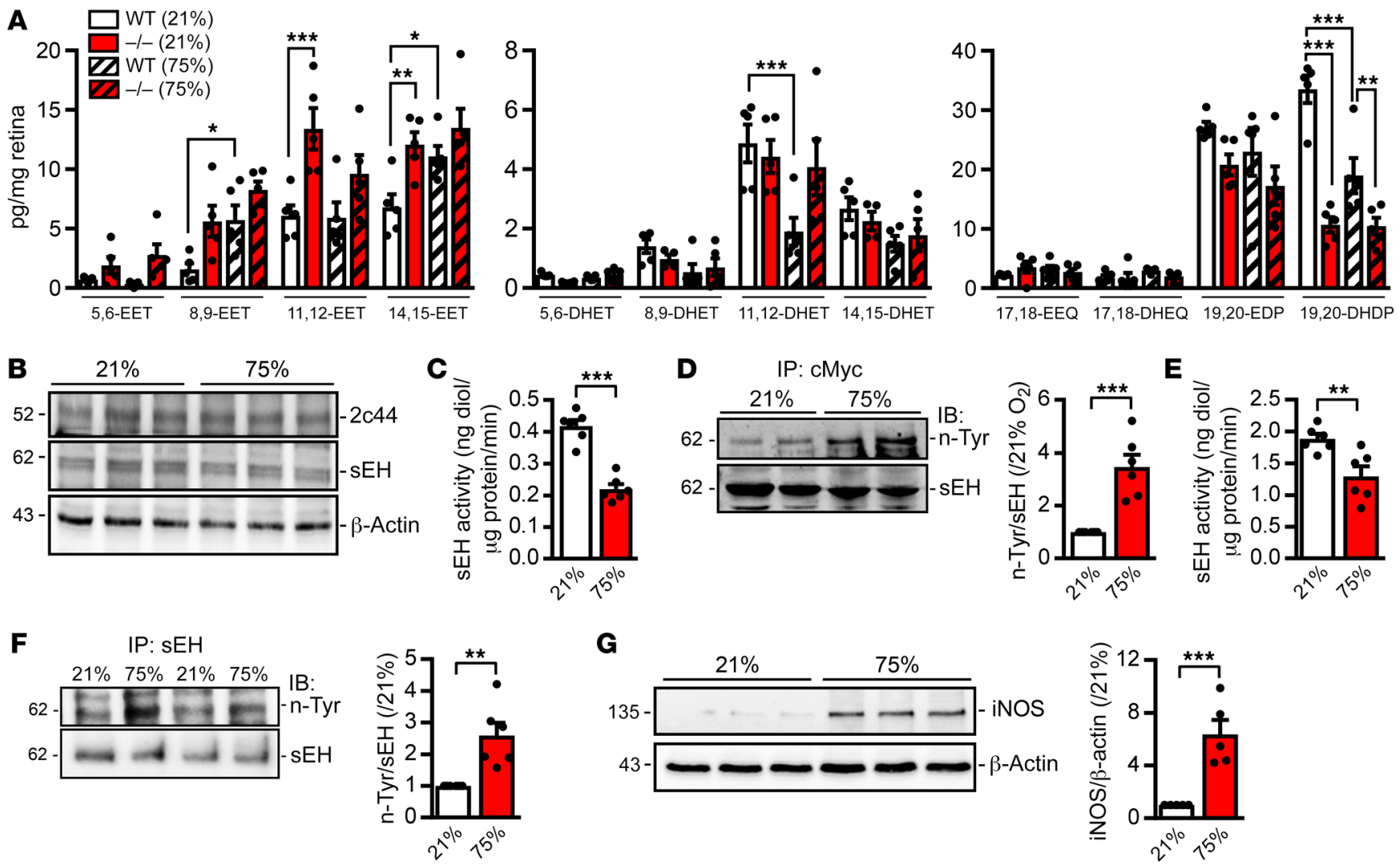

Figure 6. Consequences of hyperoxia on sEH activity. (A) Fatty acid epoxide and diol levels in retinas from WT and sEH ${ }^{-1-}$ mice exposed to normoxia (21\% $\mathrm{O}_{2}$ ) or hyperoxia $\left(75 \% \mathrm{O}_{2}\right.$ ) for 24 hours starting on P7. $n=5$ samples per group and each sample represents a pool of 6 retinas (2-way ANOVA with Tukey's multiple comparisons test). 17,18-EEQ, 17,18-epoxy eicosatetraenoic acid; 17,18-DHEQ, 17,18-dihydroxy-eicosatetraenoic acid. (B) Immunoblot showing sEH and Cyp2c44 expression in retinas from WT mice after exposure to normoxia or hyperoxia from P7 for 24 hours. Comparable results were observed in 3 additional mice per group. (C) sEH activity in retinas from WT mice after exposure to normoxia or hyperoxia from P7 for 24 hours. $n=6$ samples/group (Student's $t$ test). (D) Tyrosine nitration of sEH in sEH-cMyc expressing-HEK-293 cells exposed to $21 \% \mathrm{O}_{2}$ or $75 \% \mathrm{O}_{2}$ for 24 hours. $n=6$ independent experiments (Student's $t$ test). (E) sEH activity in sEH expressing HEK-293 cells after exposure to $21 \% \mathrm{O}_{2}$ or $75 \% \mathrm{O}_{2}$ for 24 hours. $n=6$ independent experiments (Student's $t$ test). (F) Tyrosine nitration of sEH immunoprecipitated from retinas of WT mice after exposure to normoxia or hyperoxia for 24 hours. $n=6$ animals/group (Student's $t$ test). (G) iNOS expression in retinas from WT mice after exposure to normoxia or hyperoxia for 24 hours. $n=6$ animals/group (Student's $t$ test). ${ }^{*} P<0.05,{ }^{* *} P<0.01$, and ${ }^{* *} P<0.001$. IB, immunoblot.

whether the positive actions can be attributed to direct actions of the $\omega$-3 PUFA or to DHA-derived metabolites (e.g., the epoxides and diols generated by the sequential activity of CYP enzymes and $\mathrm{sEH}$ ). Relatively little is known about the biological actions of the DHA-derived epoxides and diols, but the epoxides (EDPs) were reported to inhibit inflammation in human retinal microvascular endothelial cells (33) as well as in a mouse model of choroidal neovascularization (34) and have also been linked to increased pathological neovascularization $(14,35)$. This is relevant, as the hyperoxia-associated inhibition of $\mathrm{sEH}$ would be expected to increase levels of angiogenic epoxides derived from DHA as well as from other PUFAs, such as arachidonic acid, to contribute to the neovascularization and increased peripheral vascular tuft formation observed. However, the intravitreal administration of 19,20-EDP failed to increase neovascularization above levels seen in solvent-treated mice. In addition, although retinal levels of 11,12- and 14,15-EET were increased in $\mathrm{sEH}^{-/-}$mice and the sEH-dependent metabolism of 11,12-EET to 11,12-DHET in WT retinas was decreased by exposure to hyperoxia, there was no clear increase in retinal levels of the epoxide in the latter samples.
Given these observations, together with the fact that a low concentration of 19,20-DHDP significantly improved revascularization, we focused more on elucidating the molecular events regulated by the DHA-derived diol.

Whether the actions of DHA-derived diols can be considered protective or deleterious seems to depend on their local concentration, largely determined by the expression and activity of sEH. For example, the expression of sEH and the generation of 19,20DHDP are required in the postnatal retina for the regulation of Notch signaling and physiological angiogenesis. The deletion or inhibition of $\mathrm{sEH}$ in the postnatal retina impedes angiogenesis and retinal vascularization (15), as well as the revascularization described herein. In adults, however, markedly increased expression of $\mathrm{sEH}$, such as that detected in diabetes, is associated with a pronounced increase in 19,20-DHDP production that has been linked to the dissolution of intracellular junctions and the vascular destabilization associated with diabetic retinopathy (25) and possibly also wet age-related macular degeneration (36). The overexpression of sEH in retinal Müller cells was sufficient to elicit retinopathy in nondiabetic WT mice (25). Thus, it seems that 
A

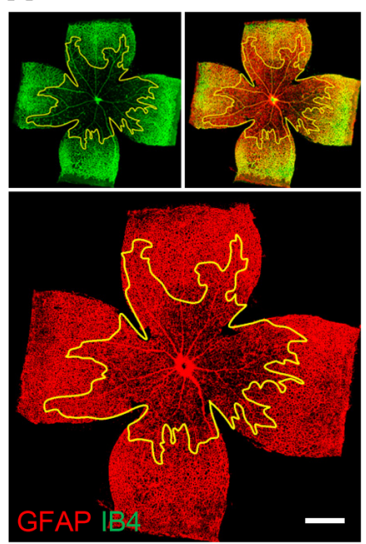

C

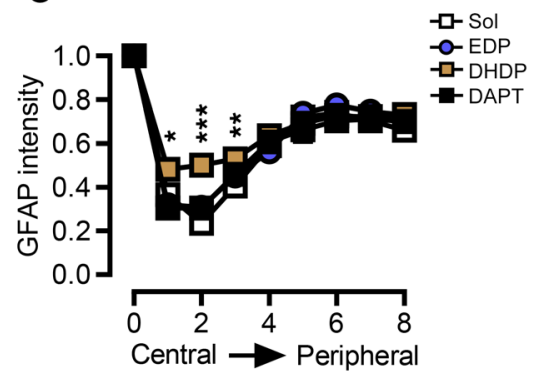

$\mathbf{F}$

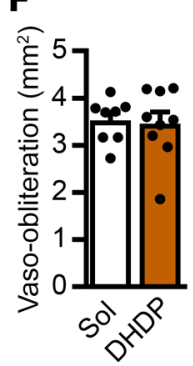

19,20-EDP
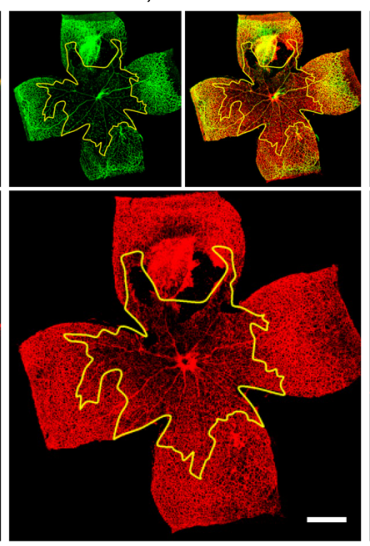

19,20-DHDP
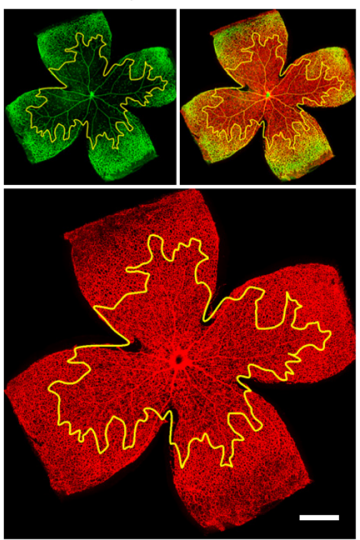

DAPT
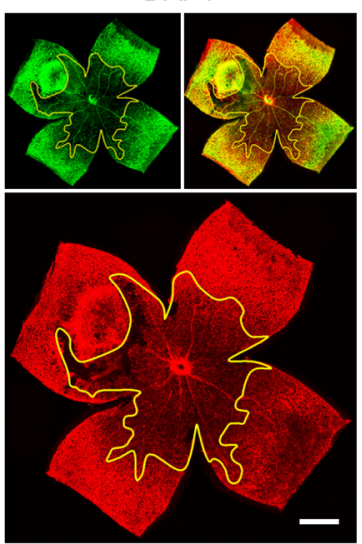

B

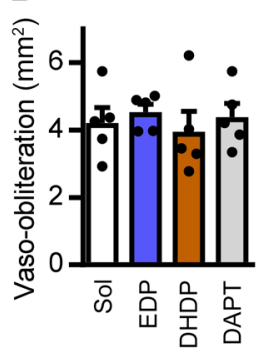

D

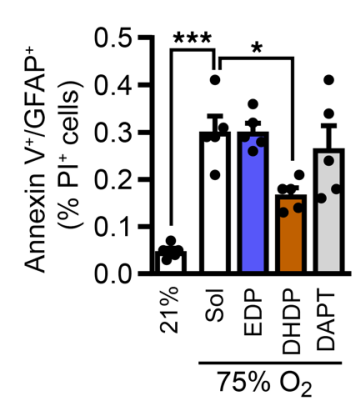

E

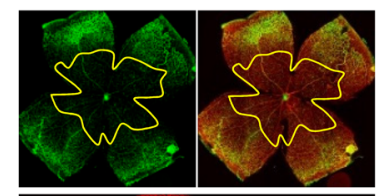

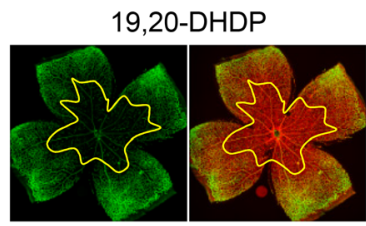
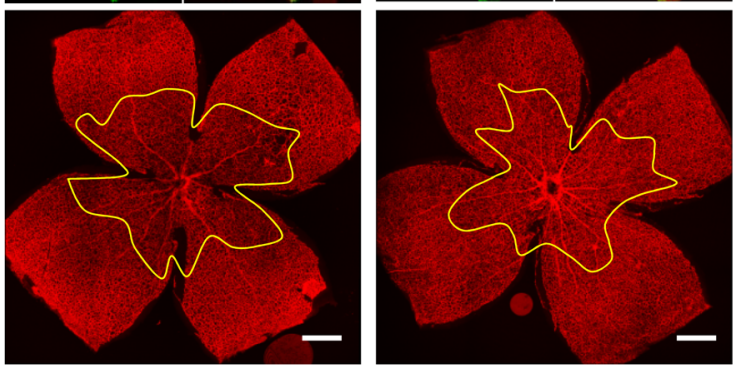

Figure 7. Effect of the sEH substrate 19,20-EDP and product 19,20-DHDP on astrocyte apoptosis. (A) $\mathrm{sEH}^{-/-}$mice were treated with a bolus of solvent (Sol) (1\% DMSO), EDP/DHDP (50 pmol), or DAPT (100 pmol) via intravitreal injection on P7 and exposed to hyperoxia for 24 hours. GFAP (red) and isolectin B4 (IB4, green) staining in retina whole mounts. Yellow lines highlight the vaso-obliterated zones in the central region of retinas. (B) Quantification of the vasoobliterated areas in $\mathrm{sEH}^{-/-}$animals exposed to hyperoxia. (C) Quantification GFAP intensity throughout the $\mathrm{sEH}^{-/-}$retina. The numbers 0 and 8 represent the optical nerve and retinal boundary, respectively (2-way ANOVA and Tukey's multiple comparisons test). (D) Quantification of annexin $\mathrm{V}^{+} / \mathrm{GFAP}^{+}$cells. (E) WT mice were treated with an intravitreal bolus of solvent (1\% DMSO) or DHDP (50 pmol) on P7 and exposed to hyperoxia for 24 hours. Retina whole mounts were stained with isolectin B4 (green) and GFAP (red). Yellow lines highlight the vaso-obliterated zones in the central region of retinas. (F) Quantification of the vaso-obliterated areas in WT animals exposed to hyperoxia. (C) Quantification GFAP intensity throughout the WT retina. (1-way ANOVA and Tukey's multiple comparisons test). $n=5$ animals/group in $\mathbf{A}-\mathbf{D}$ and $n=8-9$ per group $\mathbf{E}-\mathbf{G}$. Scale bars: $500 \mu \mathrm{m} .{ }^{*} P<0.05,{ }^{* *} P<0.01$, and ${ }^{* * *} P<0.001$.

low concentrations of 19,20-DHDP are required for the correct vascularization of the retina, whereas high concentrations induce pathological changes in the retinal vasculature. This hypothesis could be confirmed by the intravitreal injection of different concentrations of 19,20-DHDP to WT mice. In contrast to the effects of a low concentration of 19,20-DHDP, which prevented against astrocyte apoptosis as well as neovascularization, the highest concentrations studied also induced vascular abnormalities.

This investigation set out to compare the development of ROP in WT and $\mathrm{sEH}^{-/-}$mice, but it rapidly became apparent that the inhibition of sEH may in fact be a central component of the disease pathogenesis. This conclusion is based on the fact that exposing mice to hyperoxia for as little as 24 hours increased the retinal expression of iNOS and elicited the tyrosine nitration of $\mathrm{sEH}$, a posttranslational modification that was previously reported to inhibit its activity (21). Consistent with a key role for $\mathrm{SEH}$ inhibition in ROP development, exposing WT mice to hyperoxia decreased the generation of diols derived from arachidonic acid and DHA at the same time as preventing the metabolism of a spectrum of PUFA epoxides. Our findings fit well with previous reports showing increased iNOS expression (22) and tyrosine nitration (23) in mice with ROP, although in the latter studies, iNOS induction was only reported following the return of the mice to a relative hypoxic environment. Interestingly, iNOS-derived NO was 

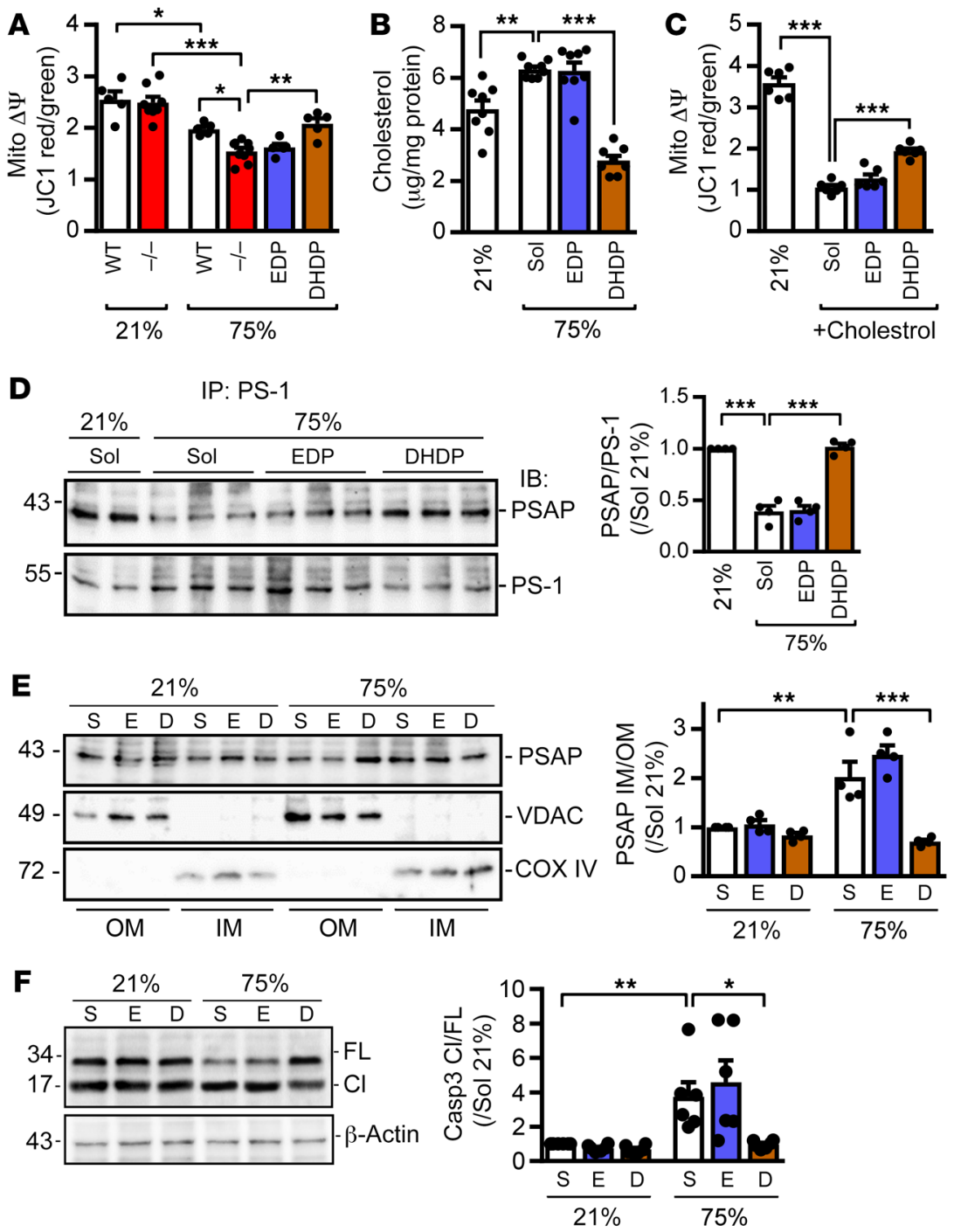

Figure 8. Effect of 19,20-DHDP on mitochondria. (A) Astrocytes from WT and $\mathrm{sEH}^{-/-}(-/-)$mice were cultured in $21 \%$ or $75 \% \mathrm{O}_{2}$ for 24 hours in the presence of solvent, 19,20-EDP, or 19,20-DHDP (3 $\mu \mathrm{M})$. Mitochondrial membrane potential (Mito $\Delta \Psi$ ) was assessed by JC-1 staining. $n=5-9$ independent isolations (1-way ANOVA and Tukey's multiple comparisons test). (B) Cholesterol levels in isolated mitochondria from HEK-293 cells treated with solvent or 19,20-EDP/DHDP $(3 \mu \mathrm{M})$ and exposed to $21 \%$ or $75 \% O_{2}$ for 24 hours. $n=8$ isolations (1-way ANOVA and Tukey's multiple comparisons test). (C) Membrane potential in isolated mitochondria after treatment with cholesterol in the absence and presence of EDP/DHDP (10 nM). $n=6-13$ independent isolations/ group (1-way ANOVA and Tukey's multiple comparisons test). (D) Immunoblot showing the association of PSAP with PS-1 immunoprecipitated from astrocytes treated with solvent or 19,20-EDP/DHDP ( $3 \mu \mathrm{M})$ and exposed to $21 \%$ or $75 \% \mathrm{O}_{2}$ for 24 hours. $n=4$ different cell batches (1-way ANOVA and Tukey's multiple comparisons test). (E) PSAP localization in the outer (OM) and inner (IM) mitochondrial membrane from HEK-293 cells exposed to $21 \%$ or $75 \% \mathrm{O}_{2}$ for 24 hours in the presence of solvent (S), 19,20-EDP (E) (3 $\mu \mathrm{M})$ or 19,20-DHDP (D) $(3 \mu \mathrm{M}) . n=4$ independent isolations (2-way ANOVA and Tukey's multiple comparisons test). (F) Full-length (FL) and cleaved (CI) caspase 3 (Casp3) in cultured retinal astrocytes exposed to $21 \%$ or $75 \% \mathrm{O}_{2}$ for 24 hours in the presence of solvent or 19,20-EDP/DHDP ( $3 \mu \mathrm{M}), n=6$ independent isolations (2-way ANOVA and Tukey's multiple comparisons test). ${ }^{*} P<0.05,{ }^{* *} P<0.01$, and ${ }^{* * *} P<0.001$. reported to inhibit angiogenesis locally in the avascular retina, and at the same time as promoting pathological intravitreal neovascularization (22), a phenotype also observed in $\mathrm{sEH}^{-/-}$mice. Therefore, it is tempting to speculate that in ROP, iNOS may exert its pathological effects at least partially through the tyrosine nitration and subsequent inhibition of sEH. Should the beneficial effects of DHA supplementation on ROP, as our data suggest, depend on the generation of 19,20-DHDP, it follows that the level of protection would be negatively correlated with the extent of tyrosine nitration of sEH. Although this would certainly be expected in the retina, it remains to be determined whether this modification can also be detected in peripheral blood from neonates receiving $\mathrm{O}_{2}$ supplementation and thus useful as a biomarker to identify subjects at high risk of disease development. In such cases, dietary supplementation of 19,20-DHDP may be a better therapeutic option than DHA supplementation, but care should be taken so that the retinal concentrations of 19,20-DHDP are sufficient to protect astrocytes and not high enough to negatively affect vascular integrity.

In the retina, $\mathrm{sEH}$ is expressed in Müller glial cells as well as in astrocytes (15), but until now, physiological angiogenesis and retinopathy have only been linked with the paracrine effects of $\mathrm{sEH}$ in Müller cells. Astrocytes clearly express sEH (37-39), however, and given the importance of the astrocyte template for endothelial cell guidance during retinal vascular development $(17,40)$ and the same process during the recovery from vaso-obliteration, it was logical to focus on the effects of 19,20-DHDP on astrocyte survival. Our observations in retinas from WT and sEH-deficient mice revealed a link between hyperoxia and astrocyte apoptosis in vivo and fit with previous work showing that increased $\mathrm{O}_{2}$ levels promote astrocyte death in vitro (41) and reduce astrocyte density in the central avascular zone of the retina in vivo (42). The profound effects of sEH on astrocyte survival rather than on vessel obliteration under hyperoxia indicate an autocrine effect of sEH in astrocytes. To confirm that the effects observed could be linked with astrocytes, experiments were repeated with mice specifically lacking sEH in GFAP-expressing cells. Although this mouse phenocopied $\mathrm{sEH}^{-/-}$mice, it should be pointed out that Müller cells can also express GFAP under certain conditions. Immunohistochemistry of retinal cross-sections indicated that the expression of GFAP in the mice studied was restricted to the first layer (consistent with astrocytes), however, rather than being evident through several retinal layers, as would be expected for Müller cells. 
A IP:PS-1
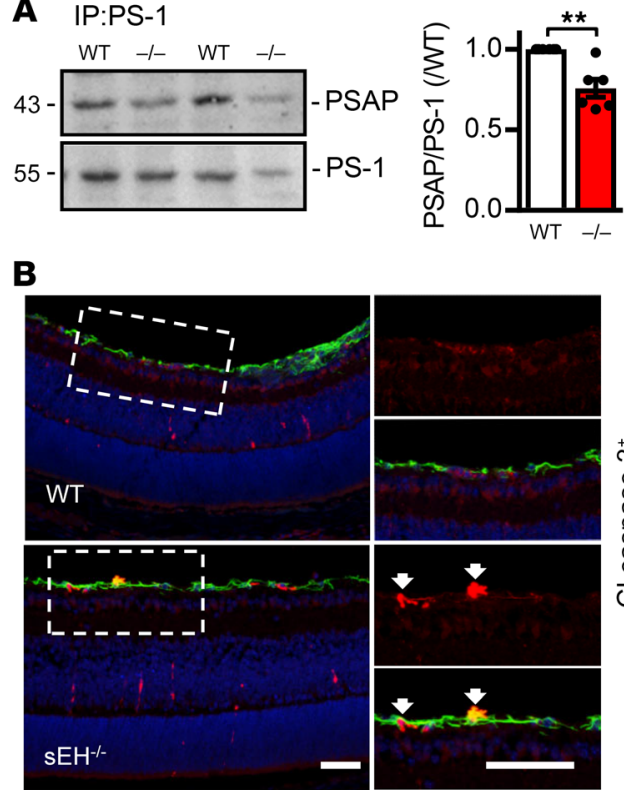

Cl caspase 3 GFAP DAPI

C IP:PS-1
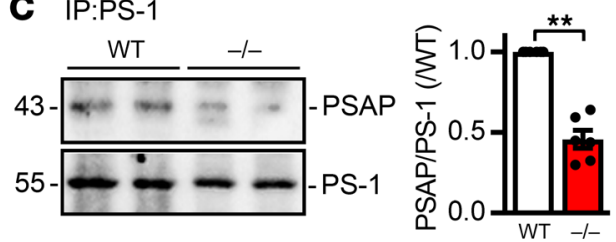
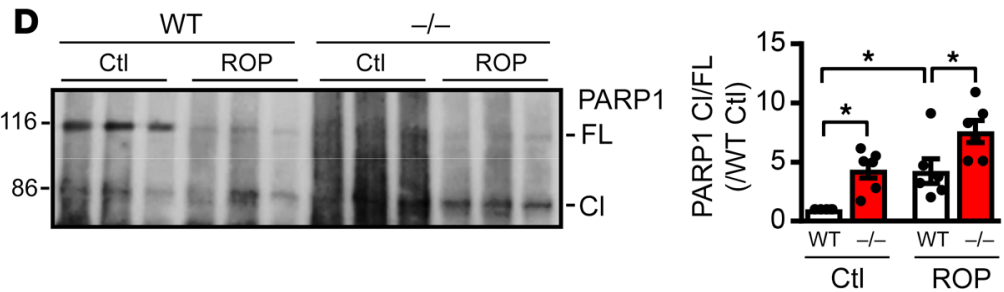
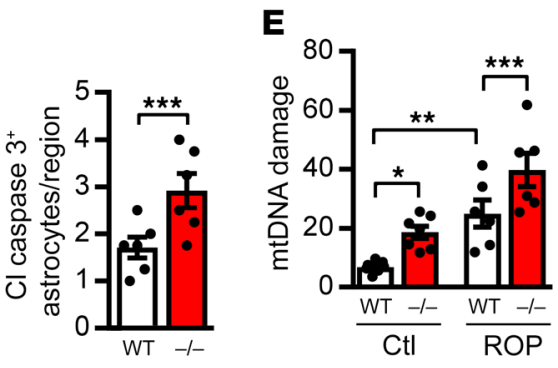

$\mathbf{F}$
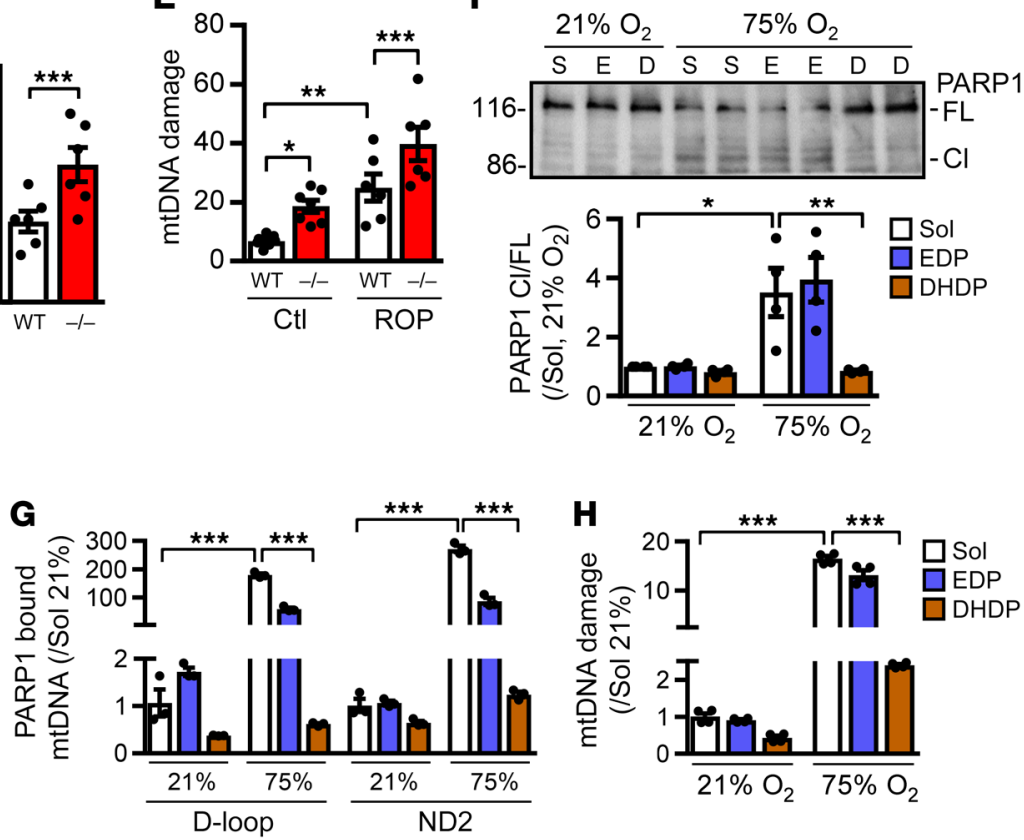

Figure 9. Link between PS-1-PARP1 and mtDNA damage. (A) Immunoblot and densitometric analysis of PSAP and PS-1 following PS-1 immunoprecipitation from retinas of WT and sEH ${ }^{-/-}(-/-)$mice exposed to hyperoxia (75\% $0_{2}$ ) for 24 hours. $n=6$ mice/group. (B) Cleaved caspase 3 (red), GFAP (green), and DAPI (blue) in cross sections from WT or $\mathrm{sEH}^{-1-}$ retinas after exposure to hyperoxia for 24 hours. $n=6$ mice/group. Scale bars: $50 \mu \mathrm{m}$. The arrows indicate GFAP cleaved caspase $3^{+}$cells. (C) Immunoblot and densitometric analysis of PSAP and PS-1 following immunoprecipitation of PS-1 in astrocytes exposed to hyperoxia for 24 hours. $n=6$ independent cell isolations. (D) Immunoblot of PARP1 in retinas from WT and sEH ${ }^{-/-}$mice. $n=6$ mice/group. (E) mtDNA damage in retinas from WT and $\mathrm{sEH}^{-1-}$ mice. $n=6-7$ mice/group. (F) Immunoblot of PARP1 in astrocytes cultured in the presence of $21 \%$ or $75 \%$ for 24 hours in the presence of solvent (0.1\% DMSO), 19,20-EDP (3 $\mu \mathrm{M})$, or 19,20-DHDP (3 $\mu \mathrm{M})$. (G) Fold enrichment of the D-Loop and ND-2 domains of mtDNA bound to the cleaved PARP1 in astrocytes exposed to $21 \%$ or $75 \% \mathrm{O}_{2}$ for 24 hours. Cells were treated with solvent or EDP/DHDP ( $\left.3 \mu \mathrm{M}\right)$. $n=3$ independent cell batches, each in duplicate. (H) mtDNA damage in astrocytes exposed to $21 \%$ or $75 \% 0$ for 24 hours in the presence of solvent or EDP/DHDP ( $3 \mu \mathrm{M}$ ). $n=4$ independent cell batches. (A-C) Student's $t$ test. (D-H) Two-way ANOVA with Tukey's multiple comparisons test. ${ }^{*} P<0.05$, ${ }^{* *} P<0.01$, and ${ }^{* * *} P<0.001$.

At the molecular level, 19,20-DHDP elicits its effects by altering membrane lipid dynamics and displacing cholesterol and cholesterol-interacting proteins from specific microdomains in the plasma membrane $(15,25)$. This unique behavior of 19,20-DHDP seems to be conserved in astrocyte mitochondrial membranes, and although it is not possible to definitively state that 19,20DHDP can be generated within or proximal to astrocyte mitochondria, it should be noted that sEH is localized to mitochondria in the liver $(43,44)$. The best-characterized cholesterol-linked protein targeted by 19,20-DHDP is PS-1, which was of particular interest, as it has also been attributed to $\gamma$-secretase-dependent and -independent proapoptotic effects in mitochondria $(27,28)$. In addition, alterations in membrane cholesterol have also been linked with mitochondrial damage and death (26). Given the lack of an effect of a $\gamma$-secretase inhibitor on astrocyte survival during the development of ROP, emphasis was placed on identifying an alternative link between PS- 1 and apoptosis. Indeed, $\gamma$-secre- tase-independent actions of PS-1 are reportedly mediated by its interaction with PSAP (28), which localizes to the mitochondrial membrane and induces apoptosis in a Bax/Bak-independent but caspase 3-dependent manner $(28,29)$. This link fits well with the observed hyperoxia-induced dissociation of PS-1/PSAP complex and the translocation of PSAP to the inner mitochondrial membrane and caspase 3 activation that were prevented by 19,20-DHDP. The caspase 3-dependent proapoptotic actions of PSAP were previously linked to the cleavage of PARP1 (29), and it was possible to demonstrate enhanced PARP1 cleavage in retinas from WT mice exposed to hyperoxia and in retinas from normoxic $\mathrm{sEH}^{-/-}$mice, as well as the enhanced binding of PARP1 to mtDNA. Again, all of these alterations were prevented by 19,20DHDP, thus identifying a molecular mechanism for its protective effect on astrocyte survival and prevention of ROP development. One clear limitation of the current investigation is that the study was limited to a single animal model, although the mouse model 
A
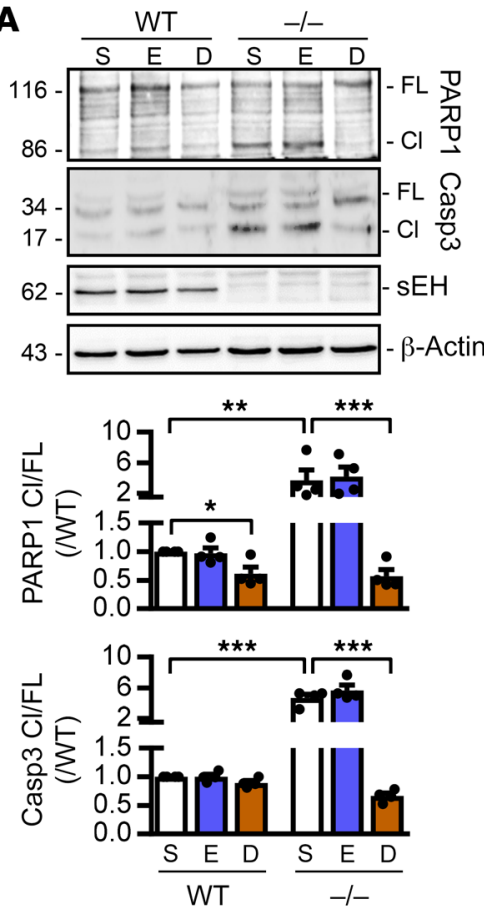

B

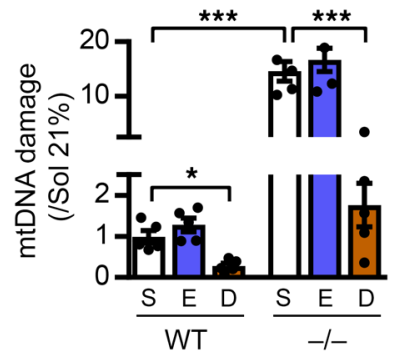

C
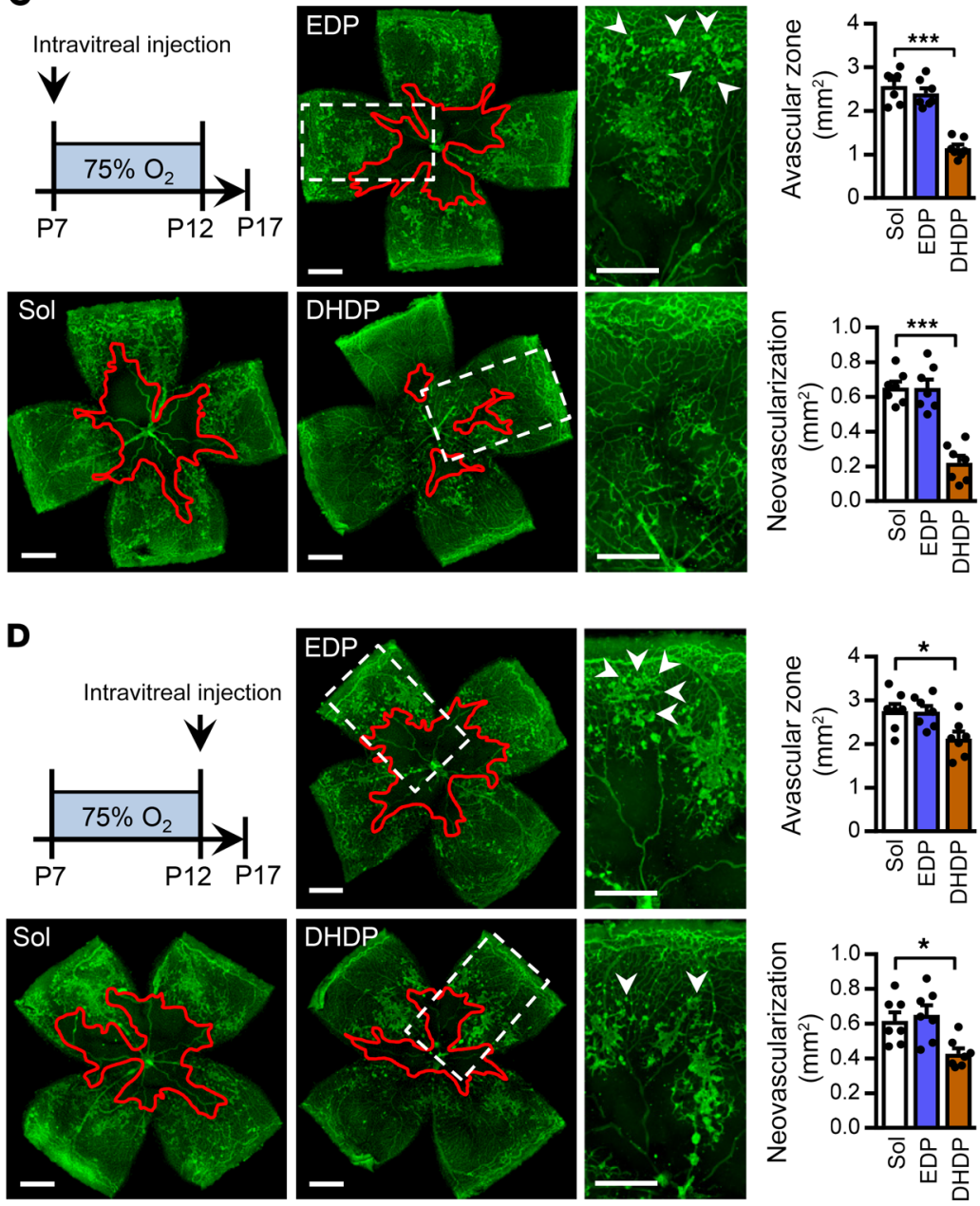

Figure 10. 19,20-DHDP prevents neovascularization in ROP. WT and $\mathrm{sEH}{ }^{-/-}$mice were treated with an intravitreal bolus of solvent (1\% DMSO), 19,20-EDP (50 pmol), or 19,20-DHDP (50 pmol) on P7 and exposed to hyperoxia for 24 hours. (A) Immunoblot showing PARP1 and caspase cleavage in retinas at P8. $n=4$ animals/group (B) mtDNA damage was quantified in retinas isolated on P8. $n=5$ animals/group. (C) $\mathrm{sEH}^{-/-}$mice were treated with solvent or 19,20EDP/DHDP (50 pmol) on P7 before being exposed to hyperoxia. Isolectin B4 staining (green) was performed on retinal whole mounts at P17. $n=7$ animals/ group. (D) $\mathrm{SEH}^{-1-}$ mice were exposed to hyperoxia for 5 days and then treated with solvent or 19,20-EDP/DHDP (50 pmol) on P12. Isolectin B4 staining (green) was performed on retinal whole mounts on P17. $n=7$ animals/group. Scale bars: $500 \mu \mathrm{m}$. Red lines highlight the border of the avascular regions. Arrows indicate peripheral neovascularization. ${ }^{*} P<0.05$, ${ }^{*} P<0.01$, and ${ }^{* * *} P<0.001$ (2-way ANOVA and Tukey's multiple comparisons test for $\mathbf{A}$ and $\mathbf{B}$ and 1-way ANOVA with Bonferroni's multiple comparisons test for $\mathbf{C}$ and $\mathbf{D})$.

studied reproduces many of the unique characteristics observed in infants with ROP. Certainly, our data indicate that defective 19,20-DHDP production underlies the pathogenesis of ROP and make it tempting to speculate that low concentrations of 19,20DHDP may protect against retinopathy in preterm infants receiving $\mathrm{O}_{2}$ supplementation.

\section{Methods}

Materials. Cell culture media were from Gibco (Invitrogen).19,20-EDP acid and 19,20-DHDP and all epoxide and diol standards for liquid chromatography-tandem mass spectrometry were obtained from Cayman Europe. The sEH inhibitor trans-4- [4-(3-adamantan-1-ylureido) cyclohexyloxy]-benzoic acid (45) was provided by Bruce D. Hammock (UCD, Davis, California, USA). OCT Tissue Tek was from Sakura and the protease inhibitor cocktail was from Roche. All other chemicals (unless otherwise specified) were from Sigma-Aldrich.
The rabbit polyclonal antibody against Cyp2c44 was provided by Darryl C. Zeldin (Triangle Park, North Carolina, USA) and the rabbit polyclonal antibody against $\mathrm{sEH}$ was provided by Michael Arand (Zurich, Switzerland). Antibodies against NG-2 (AB5320), presenilin-1 (MAB1563), and nitrotyrosine (06-284) were from Millipore. Isolectin-B4 (L2895) and anti- $\beta$-actin (A1978) were from Sigma-Aldrich. The anti-GFAP antibodies were from DAKO (Z0334, Sakura) and Millipore (AB5541). The Tom20 antibody (sc17764) and the iNOS antibody (sc-7271) were from Santa Cruz Biotechnology. Antibodies against VDAC (no. 4661), cytochrome c oxidase (COX) IV (no. 4844), caspase 3 (no. 9662), and cleaved caspase 3 (no. 9664) were from Cell Signaling Technology. The anti-PARP-1 antibody (no. 551024) was from BD Biosciences, and the anti-PSAP antibody (BS-7630R) was from Bioss Antibodies. The Fitc-Annexin V (no. 556420; BD) and GFAP-AF647 (no. sc-33673; Santa Cruz) were used for FACS analysis. Secondary antibodies for Western blotting were from Calbiochem. 
Fluorophore-conjugated secondary antibodies from confocal imaging were from Invitrogen (Alexa Fluor).

Animals and in vivo treatment. Six- to 8-week-old C57BL/6 mice were purchased from Charles River. Floxed $\mathrm{sEH}$ mice (Ephx ${ }^{\left.\mathrm{tm}^{\mathrm{m} 1.1 \mathrm{Arte}}\right)}$ were generated by TaconicArtemis $\mathrm{GmbH}$ as described (15). They were either crossed with Gt(ROSA)26Sortm16(Cre)Arte mice (TaconicArtemis) expressing Cre under the control of the endogenous Gt(ROSA)26Sor promoter to generate mice globally lacking $\mathrm{sEH}\left(\mathrm{sEH}^{-/}\right)$or with B6.Cg-Tg(Gfap-cre)77.6Mvs/2J mice (The Jackson laboratory, stock no. 024098) (46). All animals were housed in conditions that conform to the Guide for the Care and Use of Laboratory Animals published by the NIH (publication no. 85-23). Age- and strain-matched animals (littermates) of both genders were used throughout.

$R O P$. Oxygen-induced retinopathy was induced as previously described $(11,12)$. Briefly, pups were grown under room air until P7 and then exposed to $75 \% \mathrm{O}_{2}$ in a ventilated chamber (BioSpherix) with their mothers for 5 days (P7 to P12). Afterwards, they were returned to room air from P12 to P17. Age-matched control mice were kept in room air. To evaluate the short-term effects of hyperoxia on astrocytes, animals were kept in $75 \% \mathrm{O}_{2}$ for 24 hours from P7 to P8. Animals were sacrificed at the times indicated in the Results section and retinas were isolated for morphological or molecular studies.

Intravitreal injection. Intravitreal injections were performed as described (15). Briefly, pups were anesthetized with $2 \%$ isoflurane, and a drop of $0.5 \%$ proxymetacain hydrochloride (Ursapharm) was applied on top of the cornea. Each eye received $0.5 \mu \mathrm{L}$ solvent (1\% DMSO in $0.9 \% \mathrm{NaCl}), 19,20-\mathrm{EDP}(0.5 \mu \mathrm{L}$ of a $100-\mu \mathrm{M}$ solution; i.e., a bolus dose of $50 \mathrm{pmol}), 19,20-\mathrm{DHDP}(0.5 \mu \mathrm{L}$ of $30-, 100-, 300-$, or $1,000-\mu \mathrm{M}$ solution; i.e., a bolus dose of $15,50,150$, or 500 pmol) or DAPT ( 0.5 $\mu \mathrm{L}$ of a $200-\mu \mathrm{M}$ solution; i.e., a bolus dose of $100 \mathrm{pmol})$. The injections were performed using an Ultramicropump III injector (World Precision Instruments) with self-pulled glass pipettes at a rate of $10 \mathrm{~nL} / \mathrm{second}$ and continuously monitored with the aid of a stereo microscope.

Retina preparation and analysis. To determine retinal neovascularization, eyes from pups at P17 were immersed in $4 \%$ formalin for 24 hours and embedded in paraffin. Six-micrometer serial sections of whole eyes were cut sagittally through the cornea and parallel to the optic nerve and stained with periodic acid/Schiff reagent and hematoxylin. Ten sections of equal length, each $18 \mu \mathrm{m}$ apart, on both sides of the optic nerve were used to quantify retinal vascular cell nuclei on the vitreal side of the inner limiting membrane. The quantification was performed by a person blinded to the sample identity. The average number of neovascular nuclei in 10 sections was used for each eye for statistical analysis (47). For whole-mount immunohistochemistry, eyes were fixed in $4 \%$ paraformaldehyde (PFA) for 2 hours at room temperature or overnight at $4^{\circ} \mathrm{C}$. After isolation, retinas were blocked and permeabilized in 1\% BSA and 0.5\% Triton X-100 overnight at $4^{\circ} \mathrm{C}$. Then retinas were washed 3 times in Pblec buffer $(0.5 \%$ Triton X-100, 1-mM CaCl, $1-\mathrm{mM} \mathrm{MgCl}_{2}$, and $1-\mathrm{mM} \mathrm{MnCl}_{2}$ in PBS; $\mathrm{pH}, 6.8)$ and incubated overnight in Pblec containing Fitc-Isolectin B4 (1:100). The following primary antibodies were diluted in 1\% BSA and $0.5 \%$ Triton X-100 and incubated overnight: sEH (1:250), GFAP (1:500), NG-2 (1:1,000). Afterward, Alexa Fluor-coupled secondary antibodies (1:200) were used. Cell nuclei were visualized with DAPI (1:200; Molecular Probes, Thermo Fisher Scientific). Cryosections $(10-\mu \mathrm{m})$ were cut after retinas were embedded within Tissue-Tek OCT Compound. All high-resolution fluorescent images were taken using a laser scanning confocal microscope (LSM-510, LSM-780 Carl Zeiss, or TCS-SP5, Leica). Vaso-obliteration and pathological neovascularization were analyzed as described (12). Fluorescent images (GFAP) were analyzed by Axiovision software (Zeiss) to determine astrocyte areas and numbers. GFAP intensity across the retina was determined by dividing the leaves into 8 radial sections (see Figure $2 \mathrm{~B})$, where the number 0 and 8 represent the optical nerve and retinal boundary, respectively. More detailed analysis of the astrocyte network was performed by determining the percentage $\mathrm{GFAP}^{+}$area/ total area measured in "central" and "front" areas of interest, located 1-third and 2-thirds of the distance between the optic nerve and the vascular boundary, respectively. Eight areas of interest (each $400 \times$ $400 \mu \mathrm{m})$ per retina were analyzed using an automatic thresholding mode to select $\mathrm{GFAP}^{+}$area based on GFAP intensity. In the same samples, the numbers of astrocytes (i.e., $\mathrm{GFAP}^{+}$stellar cells) were counted and the data presented as number $/ \mathrm{mm}^{2}$. To determine apoptosis in the retinal whole mounts, pups were intravitreally injected with $0.5 \mu \mathrm{L}$ FITC-annexin V (no. 556420, BD) after exposure to $75 \%$ oxygen for 24 hours. After 15 minutes, the eyes were removed and fixed in $4 \%$ PFA for 2 hours at room temperature. Retinas were isolated and immunostaining against GFAP (1:500) was performed as described previously.

FACS analysis. For FACS analysis on retinal cells, pups received an intravitreal injection with $0.5 \mu \mathrm{L}$ FITC-annexin V (no. 556420, BD) after exposure to $75 \%$ oxygen for 24 hours. After 15 minutes, retinas were digested with $0.1 \%$ trypsin (Worthington Biochemical Corporation) and $70 \mathrm{U} / \mathrm{mL}$ collagenase type I (Biochrom) in DMEM/F12 medium at $37^{\circ} \mathrm{C}$ for 30 minutes. Cells were dissociated and passed through a $40-\mu \mathrm{m}$ cell strainer to reach single-cell suspension. Retinal cells were fixed and permeabilized (Fixation/Permeabilization Solution Kit, BD) and blocked for 30 minutes with the Fc-block and subsequently incubated with anti-GFAP AF647 antibody (1:200) and propidium iodide. Next, these cells were analyzed on a BD FACSVerse flow cytometer (BD). For FACS analysis on cultured astrocytes, the astrocytes were trypsinized with $0.25 \%$ trypsin (Sigma-Aldrich) and processed for annexin $\mathrm{V}$ staining according to the manufacturer's instructions (Annexin V apoptosis kit, BD). Apoptotic cells were plotted as a percentage of the annexin V and GFAP double-positive cells versus the total propidium iodide-positive cell number.

Astrocyte isolation and culture. Retinal astrocytes were isolated as described (48). In brief, 12 to 14 retinas from 4 -week-old mice were isolated under a dissecting microscope, rinsed with serum-free DMEM, and digested for 30 minutes with Collagenase Type I $(100 \mathrm{U} / \mathrm{mL}$, Biochrom, Merck) in serum-free DMEM at $37^{\circ} \mathrm{C}$. Digested cells were rinsed in DMEM containing 10\% FBS and filtered through a sterile $40-\mu \mathrm{m}$ cell strainer. Cells were washed twice with DMEM containing $10 \%$ FBS and resuspended in $1 \mathrm{~mL}$ DMEM containing 10\% FBS with rat anti-mouse CD31 clone MEC13.3 (no. 550274, BD), coated sheep anti-rat magnetic beads, and were gently rocked for 1 hour at $4^{\circ} \mathrm{C}$. Using a Dynal magnetic tube holder, cells not bound to magnetic beads were collected and washed in DMEM containing 10\% FBS. Cells were plated in fibronectin-coated $(2 \mu \mathrm{g} / \mathrm{mL}$ in serum-free DMEM; BD Biosciences) dishes and cultured in DMEM containing 10\% FBS, 2-mM L-glutamine, 2-mM sodium pyruvate, 20-mM HEPES, 1\% nonessential amino acids, $100 \mu \mathrm{g} / \mathrm{mL}$ streptomycin, $100 \mathrm{U} / \mathrm{mL}$ penicillin, $55 \mathrm{U} /$ $\mathrm{mL}$ heparin (Sigma-Aldrich), endothelial growth supplement $100 \mu \mathrm{g} /$ $\mathrm{mL}$ (Sigma-Aldrich), and the murine recombinant IFN- $\gamma$ (R\&D Systems) at $44 \mathrm{U} / \mathrm{mL}$ and incubated at $37^{\circ} \mathrm{C}$ with $5 \% \mathrm{CO}_{2}$. Experiments 
were performed using cells between passage 2 and 4, and a total of 6 to 9 biologically independent cell preparations were studied.

Caspase 3/7 activity. Retinal astrocytes (passage 2) from WT and $\mathrm{sEH}^{-/-}$mice were exposed to normoxia $\left(21 \% \mathrm{O}_{2}\right)$ or hyperoxia $(75 \%$ $\mathrm{O}_{2}$ ) for 24 hours. Cells were collected and caspase 3 and 7 activity was measured with a commercially available kit (ab39383, Abcam) according to the manufacturer's instructions.

Cell culture. HEK-293 cells were obtained from the American Type Culture Collection (LGC Standards) and cultured in MEM containing $8 \%$ heat-inactivated FCS, gentamycin $(25 \mu \mathrm{g} / \mathrm{mL})$, nonessential amino acids (MEM NEAA, Thermo Fisher Scientific), and Na pyruvate (1 mM). Cultures were kept in a humidified incubator at $37^{\circ} \mathrm{C}$ containing $5 \%$ $\mathrm{CO}_{2}$. Experiments were performed in 4 to 6 independent cell passages.

sEH tyrosine nitration. HEK-293 cells were transfected with either GFP or human WT sEH-myc tag plasmid (21) using a Lipofectamine 2000 reagent (Thermo Fisher Scientific) according to the manufacturer's instructions. Twenty-four hours after transfaction, cells were lysed in $50 \mathrm{mM}$ Tris/ $\mathrm{HCl}$ (pH 7.5), $50 \mathrm{mM} \mathrm{NaCl}, 10 \mathrm{mM} \mathrm{NaF}, 1 \%$ sodium deoxycholate, and $1 \%$ Triton X-100 buffer. sEH was immunoprecipitated by using a Myc-resin antibody (Santa Cruz Biotechnology). Proteins enriched were separated by SDS-PAGE and immunoblotted against nitrotyrosine (1:1000) and sEH (1:1000). sEH tyrosine nitration in retinas was detected following lysis retinas in the same buffer and immunoprecipitation of sEH using a rabbit polyclonal antibody against sEH.

Mitochondria isolation and membrane fractionation. HEK-293 cells were incubated with solvent (0.1\% DMSO) or $3 \mu \mathrm{M} \mathrm{19,20-DHDP} \mathrm{in}$ serum-free medium and cultured in incubators supplied with $21 \%$ or $75 \% \mathrm{O}_{2}$ with $5 \% \mathrm{CO}_{2}$ at $37^{\circ} \mathrm{C}$ for 24 hours. Cells were then washed twice with ice-cold PBS, harvested by scraping, recovered by centrifugation (300 $g$ for 4 minutes). Mitochondria were isolated using the mitochondria isolation kit for mammalian cells according to the manufacturer's instructions (Thermo Fisher Scientific). Isolated mitochondria were resuspended in $2 \mathrm{~mL}$ MBS buffer (containing 1\% CHAPSO in $25 \mathrm{mM}$ MES (pH 6.5), $150 \mu \mathrm{M} \mathrm{NaCl}$ supplemented with a protease inhibitor mix, and $1 \mu \mathrm{M}$ phenylmethylsulfonyl fluoride), and homogenized by using an ultrasound homogenizer. Mitochondria membrane fractions were isolated as previously described for plasma membranes (49). In brief, after 30 minutes at $4^{\circ} \mathrm{C}$, equal amounts of protein were adjusted to a final sucrose concentration of $45 \%$ (final volume, $4 \mathrm{~mL}$ ) and transferred to $12-\mathrm{mL}$ ultracentrifuge tubes. A discontinuous sucrose gradient was then formed by sequentially overlaying $4 \mathrm{~mL}$ of $35 \%$ and $4 \mathrm{~mL}$ of $5 \%$ sucrose. Samples were subjected to ultracentrifugation $(35,000$ $\mathrm{rpm}, 4^{\circ} \mathrm{C}$ for 20 hours) using a rotor (SW 41, Beckman Coulter). After centrifugation, twelve 1-mL fractions were collected using a capillary tube connected to a peristaltic pump. Equal volumes of each faction were precipitated with $20 \%$ trichloroacetic acid and washed twice with cold acetone and recovered in Tris-buffered saline and analyzed by SDS-PAGE. For isolation of the outer and inner mitochondrial membrane, isolated mitochondrial pellets were resuspended in $1.7 \mathrm{~mL}$ of $0.1-\mathrm{M} \mathrm{Na}_{2} \mathrm{CO}_{3}(\mathrm{pH} 12)$ and incubated on ice for 20 minutes. Subsequently, they were centrifuged at $144,000 \mathrm{~g}$ for 1 hour at $4^{\circ} \mathrm{C}$. The supernatant contained the outer membrane fragments. The pellet enriched in inner mitochondrial membrane proteins was resuspended using a Pasteur pipette in PBS supplemented with $1 \%$ Triton-X 100 followed by repeated passage through a 20-gauge and then a 27-gauge needle (50).

Cholesterol incorporation assay. HEK-293 cells were treated with solvent (0.1\% DMSO), EDP $(3 \mu \mathrm{M})$, DHDP $(3 \mu \mathrm{M})$, and cultured under $21 \%$ or $75 \% \mathrm{O}_{2}$ in a humidified chamber at $37^{\circ} \mathrm{C}$ for 24 hours. Afterward, mitochondria were isolated as described previously, and the levels of cholesterol in the isolated mitochondria were measured using the Amplex Red cholesterol assay (Invitrogen). For the cholesterol feeding experiment, mitochondria were isolated from HEK-293 cells and were then pretreated with solvent (0.0001\% DMSO), EDP $(10 \mathrm{nM})$, or DHDP $(10 \mathrm{nM})$ for 10 minutes. Next, mitochondria were treated with a cholesterol-BSA complex for 10 minutes in room temperature as described (51).

sEH activity assay. Retinas or HEK-293 cells overexpressing either GFP or the human WT sEH were homogenized in RIPA lysis buffer (50 mM Tris/HCl [pH 7.5], $150 \mathrm{mM} \mathrm{NaCl}, 10 \mathrm{mM} \mathrm{NaPPi}, 20 \mathrm{mM} \mathrm{NaF}$, $1 \%$ sodium deoxycholate, $1 \%$ Triton X-100, and $0.05 \%$ SDS) enriched with protease and phosphatase inhibitors, and the homogenate was used to determine $\mathrm{SEH}$ activity. Briefly, reactions were performed with $5 \mu \mathrm{g}$ protein at $37^{\circ} \mathrm{C}$ for 10 minutes in $100 \mu \mathrm{L}$ potassium phosphate buffer (100 mM; pH 7.2). Reactions were started by the addition of 10 $\mu \mathrm{M}$ 14,15-EET, stopped on ice, and immediately extracted twice with ethyl acetate $(0.7 \mathrm{~mL})$. For liquid chromatography-tandem mass spectrometry analysis, one-tenth of the sample was spiked with a deuterated internal standard (14,15-EET-d8). After evaporation of the solvent in a vacuum block under a gentle stream of nitrogen, the residues were reconstituted with $50 \mu \mathrm{L}$ methanol/water (1:1, v/v) and determined with a Sciex API4000 mass spectrometer operating in multiple-reaction monitoring mode. Chromatographic separation was performed on a Gemini C18 column (150 $\mathrm{mm} \times 2 \mathrm{~mm}$ inner diameter [ID], $5-\mu \mathrm{m}$ particle size; Phenomenex).

Epoxide/diol profiling. Mouse retinae were mixed with $100 \mu \mathrm{L}$ potassium phosphate buffer (100 mM;pH, 7.2) and underwent 3 freezethaw-vortex cycles. Samples were spiked with a deuterated internal standard mix (8,9-DHET-d11, 11,12-DHET-d11, 14,15-DHET-d11, 9,10-DiHOME-d4，12,13-DiHOME-d4， 5,6-EET-d11， 8,9-EET-d8, 11,12-EET-d8, 14,15-EET-d8, 9,10-EpOME-d4, 12,13-EpOME-d4, 5SHETE-d8, 12S-HETE-d8, 15S-HETE-d8, 20-HETE-d6, 9S-HODE-d4, and 13S-HODE-d4) and $500 \mu \mathrm{L}$ ethyl acetate was added. Samples were vortexed, placed on ice for 10 minutes, vortexed again, and subsequently centrifuged $\left(10,000 \mathrm{~g}, 5 \mathrm{~min}, 4^{\circ} \mathrm{C}\right)$. The upper phase was collected and the ethyl acetate extraction was performed again. Both upper-phase samples were combined and evaporated to dryness in a vacuum manifold under a continuous nitrogen stream. The residues were reconstituted with $50 \mu \mathrm{L}$ methanol/water (50:50, v/v, containing $100 \mathrm{ng} / \mathrm{mL}$ flufenamic acid as internal control) and determined with a Sciex QTrap5500 mass spectrometer operating in multiplereaction monitoring mode in the negative ionization mode. The following electrospray ionization parameters were set: curtain gas (CUR), $24 \mathrm{psi}$; ionization voltage (IS), $-4500 \mathrm{~V}$; temperature (TEM), $600^{\circ} \mathrm{C}$; gas flow 1 (GS1), 45 psi; and gas flow 2 (GS2), 60 psi. Chromatographic separation was performed on an Agilent 1290 Infinity LC system using a Gemini C18 column (150 mm $\times 2 \mathrm{~mm}$ ID, $5-\mu \mathrm{m}$ particle size; Phenomenex). The mobile phase consisted of (A) water plus $0.0125 \%$ ammonia and (B) acetonitrile plus $0.0125 \%$ ammonia. Elution of analytes was carried out under gradient conditions at a flow rate of 0.5 $\mathrm{mL} / \mathrm{min}$ going from $15 \% \mathrm{~B}$ to $40 \% \mathrm{~B}$ in 10 minutes, increasing to $90 \%$ $\mathrm{B}$ in 2 minutes, hold $90 \% \mathrm{~B}$ for 1 minute, and equilibrated in $15 \% \mathrm{~B}$ for 5.5 minutes. Ten microliters of each sample was injected onto the column. The column temperature was kept at $40^{\circ} \mathrm{C}$. Samples were kept in the autosampler at $6^{\circ} \mathrm{C}$ until analysis. Calibration curves were 
performed with authentic standards. All samples and dilutions of the standards were spiked with internal deuterated standards. Metabolite concentrations were determined by reference to the standards. Analyst 1.6.2 and MultiQuant 3.0 (both from Sciex) were used for data acquisition and analysis, respectively.

Immunoblotting. Retinas or cells were lysed in RIPA lysis buffer (50 mM Tris/HCl [pH 7.5], $150 \mathrm{mM} \mathrm{NaCl}, 10 \mathrm{mM} \mathrm{NaPPi}, 20 \mathrm{mM}$ $\mathrm{NaF}$, and $1 \%$ sodium deoxycholate). One percent Triton and $0.1 \%$ SDS enriched with protease and phosphatase inhibitors and detergent-soluble proteins were resuspended in SDS-PAGE sample buffer. Samples were separated by SDS-PAGE and subjected to Western blotting as described (52). Membranes were blocked in 3\% BSA in PBS, incubated with primary and HRP-conjugated secondary antibodies in blocking solution, and detection was performed with a Lumi-Light plus Western blotting substrate (Roche). Antibodies against 2C44 (1:1000), sEH (1:1000), PSAP (1:100), PS-1 (1:1000), VDAC (1:1000), COX IV (1:1000), PARP1 (1:1000), caspase 3 (1:1000), and $\beta$-actin (1:5000) were used. Secondary antibodies were used at a dilution of 1:10,000.

For immunoprecipitation studies, samples were lysed in $50 \mathrm{mM}$ Tris/ $\mathrm{HCl}$ (pH 7.5), $50 \mathrm{mM} \mathrm{NaCl}, 10 \mathrm{mM} \mathrm{NaF}, 1 \%$ sodium deoxycholate, and 1\% Triton X-100 buffer. Equal amounts of protein were incubated overnight with the appropriate antibodies.

CHIP-qPCR. Chromatin immunoprecipitation was performed using a Magna chip protein A/G beads kit from Millipore (Merck). Cells or retinas were fixed in $0.1 \%$ PFA. Nulceus was isolated and shear chromatin was immunoprecipitated with a PARP1 cleaved antibody (ab32064, Abcam). After elution, the binding of PARP1 to selective mitochondria DNA was monitored via qPCR.

mtDNA damage. mtDNA fragment expression levels or mtDNA interacting with PARP1 were detected using SYBR Green (Absolute QPCR SYBR Green Mix; Thermo Fisher Scientific). The integrity of the mtDNA was assessed using 2 primer pairs with amplicons of 211 bp (mtDNAfr) and $8.9 \mathrm{~kb}$. (CONmtDNA-short amplicon was used as a normalization factor of mtDNA copies.) (53). The relative expression levels of the different genes studied was calculated using the formula $2^{-\Delta C \mathrm{t}}\left[\Delta C_{\mathrm{t}}=C_{\mathrm{t} \text { (fragment) }}-C_{\mathrm{t}(\mathrm{CON})}\right]$. COX II was used as an additional control to monitor the isolation procedure. The primer sequences used were as follows: m117-mtDNAfr (forward, 5'-CCCAGCTACTACCATCATTCAAGT-3', reverse, 5'-GATGGTTTGGGAGATTGGTTGATGT-3'), CONmtDNA (forward, 5'-GCCAGCCTCTCCTGATTTTAGTGT-3', reverse, 5'-GGGAACACAAAAGACCTCTTCTGG-3'), 284-D-Loop (forward, $5^{\prime}$-CCCCTCACCCACTAGGATAC-3', reverse, 5'-ACGTGTGGGCTATTTAGGC-3'), 225-ND2 (forward, 5'-GCCCTAGAAATAAACATGCTA-3', reverse, 5'-GGGCTATTCCTAGTTTTATT-3'), 157COX II (forward, 5'-CACCTACСТCССТCACCAAA-3', reverse, 5'GGGATCAATAGAGGGGGAAA-3').

Statistics. Data are expressed as mean \pm SEM. Statistical evaluation was performed using GraphPad Prism 7.04 (GraphPad Software) using Student's $t$ test for unpaired data, 1-way ANOVA followed by Bonferroni's or Tukey's multiple comparisons test, or 2-way ANOVA with a Tukey's or Sidak's post-test where appropriate. Statistical tests are described in the figure legend for each experiment. $P<0.05$ was considered statistically significant.

Study approval. All animal experiments were approved by the governmental authorities (Regierungspräsidium Darmstadt: F28/06, F28/23, F28/38, and FU-1225).

\section{Author contributions}

JH and SIB share first authorship. JH is listed before SIB, as he initiated the study, which was then continued as a partnership. $\mathrm{JH}$ initiated the experimental work including all mouse studies and remained involved throughout. SIB performed essential biochemical analyses to define the link to astrocyte apoptosis. JH, SIB, and IF designed the experiments. JH, SIB, JW, and RP performed the experiments and analyzed data. SZ generated the epoxide and diol profiles and sEH activity assay. JL and HPH generated and characterized the retinal periodic acid Schiff and hematoxylin staining preparations. JH and IF wrote the manuscript.

\section{Acknowledgments}

The authors are indebted to Isabel Winter, Katharina Bruch, and Katharina Engel-Herbig for expert technical assistance and to Xufang Sun and Yuanjun Qin (Tongji Hospital, Wuhan, China) for critical discussions. This work was supported by the Deutsche Forschungsgemeinschaft (SFB 834/3 B13, project ID: 75732319, GRK 880 and Exzellenzcluster 2026 "Cardio-Pulmonary Institute," Project ID: 390649896).

Address correspondence to: Ingrid Fleming, Institute for Vascular Signalling, Centre for Molecular Medicine, Goethe University, Theodor Stern Kai 7, 60596 Frankfurt am Main, Frankfurt, Germany. Phone: 49.69.6301.6972; Email: fleming@em.uni-frankfurt.de.
1. Rivera JC, et al. Retinopathy of prematurity: inflammation, choroidal degeneration, and novel promising therapeutic strategies. J Neuroinflammation. 2017;14(1):165.

2. Sternberg P, Durrani AK. Evolving concepts in the management of retinopathy of prematurity. Am JOphthalmol. 2018;186:xxiii-xxxii.

3. Chan-Ling T, Gole GA, Quinn GE, Adamson SJ, Darlow BA. Pathophysiology, screening and treatment of ROP: a multi-disciplinary perspective. Prog Retin Eye Res. 2018;62:77-119.

4. Lapillonne A, Moltu SJ. Long-chain polyunsaturated fatty acids and clinical outcomes of preterm infants. Ann Nutr Metab. 2016;69(suppl 1):35-44.

5. Arterburn LM, Hall EB, Oken H. Distribution, interconversion, and dose response of n-3 fatty acids in humans. Am JClin Nutr. 2006;83(6 sup-
pl):1467S-1476S.

6. Hård AL, Smith LE, Hellström A. Nutrition, insulin-like growth factor-1 and retinopathy of prematurity. Semin Fetal Neonatal Med. 2013;18(3):136-142.

7. Fliesler SJ, Anderson RE. Chemistry and metabolism of lipids in the vertebrate retina. Prog Lipid Res. 1983;22(2):79-131.

8. Pawlik D, Lauterbach R, Walczak M, Hurkała J, Sherman MP. Fish-oil fat emulsion supplementation reduces the risk of retinopathy in very low birth weight infants: a prospective, randomized study. JPEN J Parenter Enteral Nutr. 2014;38(6):711-716.

9. Fu Z, et al. Dietary $\omega-3$ polyunsaturated fatty acids decrease retinal neovascularization by adipose-endoplasmic reticulum stress reduc- tion to increase adiponectin. Am JClin Nutr. 2015;101(4):879-888.

10. Stahl A, Chen J, Joyal JS, Smith LEH. The mouse model of oxygen-induced retinopathy (OIR). In: Zudaire E, Cuttitta F, eds. Textbook of Angiogenesis and Lymphangiogenesis: Methods and Applications. Dordrecht, Netherlands: Springer Netherlands; 2012:181-188.

11. Smith LE, et al. Oxygen-induced retinopathy in the mouse. Invest Ophthalmol Vis Sci. 1994;35(1):101-111.

12. Connor KM, et al. Quantification of oxygeninduced retinopathy in the mouse: a model of vessel loss, vessel regrowth and pathological angiogenesis. Nat Protoc. 2009;4(11):1565-1573.

13. Stahl A, et al. Short communication: PPAR $\gamma$ mediates a direct antiangiogenic effect of omega 
3-PUFAs in proliferative retinopathy. Circ Res. 2010;107(4):495-500.

14. Gong Y, et al. Cytochrome P450 oxidase $2 \mathrm{C}$ inhibition adds to $\omega$-3 long-chain polyunsaturated fatty acids protection against retinal and choroidal neovascularization. Arterioscler Thromb Vasc Biol. 2016;36(9):1919-1927.

15. Hu J, et al. Müller glia cells regulate Notch signaling and retinal angiogenesis via the generation of 19,20-dihydroxydocosapentaenoic acid. J Exp Med. 2014;211(2):281-295.

16. Hu J, et al. Role of Müller cell cytochrome P450 2c44 in murine retinal angiogenesis. Prostaglandins Other Lipid Mediat. 2017;133:93-102.

17. Dorrell MI, et al. Maintaining retinal astrocytes normalizes revascularization and prevents vascular pathology associated with oxygen-induced retinopathy. Glia. 2010;58(1):43-54.

18. Chan-Ling T, Stone J. Degeneration of astrocytes in feline retinopathy of prematurity causes failure of the blood-retinal barrier. Invest Ophthalmol Vis Sci. 1992;33(7):2148-2159.

19. Weidemann A, et al. Astrocyte hypoxic response is essential for pathological but not developmental angiogenesis of the retina. Glia. 2010;58(10):1177-1185.

20. Yamada T, Morisseau C, Maxwell JE, Argiriadi MA, Christianson DW, Hammock BD. Biochemical evidence for the involvement of tyrosine in epoxide activation during the catalytic cycle of epoxide hydrolase. J Biol Chem. 2000;275(30):23082-23088.

21. Barbosa-Sicard E, et al. Inhibition of the soluble epoxide hydrolase by tyrosine nitration. J Biol Chem. 2009;284(41):28156-28163.

22. Sennlaub F, Courtois Y, Goureau O. Inducible nitric oxide synthase mediates the change from retinal to vitreal neovascularization in ischemic retinopathy. JClin Invest. 2001;107(6):717-725.

23. Sennlaub F, Courtois Y, Goureau O. Inducible nitric oxide synthase mediates retinal apoptosis in ischemic proliferative retinopathy. J Neurosci. 2002;22(10):3987-3993.

24. Kroemer G, Galluzzi L, Brenner C. Mitochondrial membrane permeabilization in cell death. Physiol Rev. 2007;87(1):99-163.

25. Hu J, et al. Inhibition of soluble epoxide hydrolase prevents diabetic retinopathy. Nature. 2017;552(7684):248-252.

26. García-Ruiz C, Ribas V, Baulies A, FernándezCheca JC. Mitochondrial cholesterol and the Paradox in cell death. Handb Exp Pharmacol. 2017;240:189-210.

27. Xu X, et al. The novel presenilin-1-associated protein is a proapoptotic mitochondrial protein.
J Biol Chem. 2002;277(50):48913-48922.

28. Zeng L, Hu C, Zhang F, Xu DC, Cui MZ, Xu X. Cellular FLICE-like inhibitory protein (c-FLIP) and PS1-associated protein (PSAP) mediate presenilin 1-induced $\gamma$-secretase-dependent and -independent apoptosis, respectively. J Biol Chem. 2015;290(30):18269-18280.

29. Li T, Zeng L, Gao W, Cui MZ, Fu X, Xu X. PSAP induces a unique Apaf- 1 and Smac-dependent mitochondrial apoptotic pathway independent of Bcl-2 family proteins. Biochim Biophys Acta. 2013;1832(3):453-474.

30. Brunyanszki A, Szczesny B, Virág L, Szabo C. Mitochondrial poly(ADP-ribose) polymerase: the Wizard of Oz at work. Free Radic Biol Med. 2016;100:257-270.

31. Deng $Q$, et al. Dietary supplementation with omega-3 polyunsaturated fatty acid-rich oils protects against visible-light-induced retinal damage in vivo. Food Funct. 2018;9(4):2469-2479.

32. SanGiovanni JP, Chew EY. The role of omega-3 long-chain polyunsaturated fatty acids in health and disease of the retina. Prog Retin Eye Res. 2005;24(1):87-138.

33. Capozzi ME, Hammer SS, McCollum GW, Penn JS. Epoxygenated fatty acids inhibit retinal vascular inflammation. Sci Rep. 2016;6:39211.

34. Hasegawa E, et al. Cytochrome P450 monooxygenase lipid metabolites are significant second messengers in the resolution of choroidal neovascularization. Proc Natl Acad Sci U S A. 2017;114(36):E7545-E7553.

35. Shao Z, et al. Cytochrome P450 2C8 $\omega 3$-longchain polyunsaturated fatty acid metabolites increase mouse retinal pathologic neovascularization - brief report. Arterioscler Thromb Vasc Biol. 2014;34(3):581-586.

36. Sulaiman RS, et al. Chemical proteomics reveals soluble epoxide hydrolase as a therapeutic target for ocular neovascularization. ACS Chem Biol. 2018;13(1):45-52.

37. Sura P, Sura R, Enayetallah AE, Grant DF. Distribution and expression of soluble epoxide hydrolase in human brain. JHistochem Cytochem. 2008;56(6):551-559.

38. Rawal S, Morisseau C, Hammock BD, Shivachar AC. Differential subcellular distribution and colocalization of the microsomal and soluble epoxide hydrolases in cultured neonatal rat brain cortical astrocytes. JNeurosci Res. 2009;87(1):218-227.

39. Iliff JJ, Wang R, Zeldin DC, Alkayed NJ. Epoxyeicosanoids as mediators of neurogenic vasodilation in cerebral vessels. Am J Physiol Heart Circ Physiol. 2009;296(5):H1352-H1363.

40. O'Sullivan ML, et al. Astrocytes follow gan- glion cell axons to establish an angiogenic template during retinal development. Glia. 2017;65(10):1697-1716.

41. Danilov CA, Fiskum G. Hyperoxia promotes astrocyte cell death after oxygen and glucose deprivation. Glia. 2008;56(7):801-808.

42. Bucher F, Stahl A, Agostini HT, Martin G. Hyperoxia causes reduced density of retinal astrocytes in the central avascular zone in the mouse model of oxygen-induced retinopathy. Mol Cell Neurosci. 2013;56:225-233.

43. Gill SS, Hammock BD. Epoxide hydrolase activity in the mitochondrial fraction of mouse liver. Nature. 1981;291(5811):167-168.

44. Gill SS, Hammock BD. Epoxide hydrolase activity in the mitochondrial and submitochondrial fractions of mouse liver. Biochem Pharmacol. 1981;30(15):2111-2120.

45. Morisseau C, Goodrow MH, Newman JW, Wheelock CE, Dowdy DL, Hammock BD. Structural refinement of inhibitors of urea-based soluble epoxide hydrolases. Biochem Pharmacol. 2002;63(9):1599-1608.

46. Gregorian C, et al. Pten deletion in adult neural stem/progenitor cells enhances constitutive neurogenesis. J Neurosci. 2009;29(6):1874-1886.

47. Hammes HP, Brownlee M, Jonczyk A, Sutter A, Preissner KT. Subcutaneous injection of a cyclic peptide antagonist of vitronectin receptor-type integrins inhibits retinal neovascularization. Nat Med. 1996;2(5):529-533.

48. Scheef E, Wang S, Sorenson CM, Sheibani N. Isolation and characterization of murine retinal astrocytes. Mol Vis. 2005;11:613-624.

49. Vetrivel KS, et al. Association of gamma-secretase with lipid rafts in post-Golgi and endosome membranes. J Biol Chem. 2004;279(43):44945-44954.

50. Lamarca V, et al. Two isoforms of PSAP/MTCH1 share two proapoptotic domains and multiple internal signals for import into the mitochondrial outer membrane. Am J Physiol Cell Physiol. 2007;293(4):C1347-C1361.

51. Martínez F, Eschegoyen S, Briones R, Cuellar A. Cholesterol increase in mitochondria: a new method of cholesterol incorporation. J Lipid Res. 1988;29(8):1005-1011.

52. Fleming I, Fisslthaler B, Dixit M, Busse R. Role of PECAM-1 in the shear-stress-induced activation of Akt and the endothelial nitric oxide synthase (eNOS) in endothelial cells. JCell Sci. 2005;118(pt 18):4103-4111.

53. Szczesny B, et al. Mitochondrial DNA damage and subsequent activation of Z-DNA binding protein 1 links oxidative stress to inflammation in epithelial cells. Sci Rep. 2018;8(1):914. 